\title{
A comparison of predicted and observed ocean tidal loading in Alaska
}

\author{
H.R. Martens ${ }^{\oplus 1}$ and M. Simons ${ }^{2}$ \\ ${ }^{1}$ Department of Geosciences, University of Montana, Missoula, MT 59812, USA. E-mail: hilary.martens@umontana.edu \\ ${ }^{2}$ Seismological Laboratory, Division of Geological and Planetary Sciences, California Institute of Technology, Pasadena, CA 91125, USA
}

Accepted 2020 June 24. Received 2020 June 24; in original form 2019 September 20

\section{SUMMAR Y}

We investigate the elastic and anelastic response of the crust and upper mantle across Alaska to mass loading by ocean tides. GPS-inferred surface displacements recorded by the Plate Boundary Observatory network are compared with predictions of deformation associated with the redistribution of ocean water due to the tides. We process more than $5 \mathrm{yr}$ of GPS data from 131 stations using a kinematic precise point positioning algorithm and estimate tidal contributions using harmonic analysis. We also forward calculate load-induced surface displacements by convolving ocean-tide models with load Green's functions derived from spherically symmetric Earth models. We make the comparisons for dominant tidal harmonics in three frequency bands: semidiurnal $\left(\mathrm{M}_{2}\right)$, diurnal $\left(\mathrm{O}_{1}\right)$ and fortnightly $\left(\mathrm{M}_{\mathrm{f}}\right)$. Vector differences between predicted and observed ocean tidal loading (OTL) displacements are predominantly sub-mm in magnitude in all three frequency bands and spatial components across the network, with larger residuals of up to several $\mathrm{mm}$ in some coastal areas. Accounting for the effects of anelastic dispersion in the upper mantle using estimates of $Q$ from standard Earth models reduces the residuals for the $\mathrm{M}_{2}$ harmonic by an average of $0.1-0.2 \mathrm{~mm}$ across the network and by more than $1 \mathrm{~mm}$ at some individual stations. For the relatively small $\mathrm{M}_{\mathrm{f}}$ tide, the effects of anelastic dispersion $(<0.03 \mathrm{~mm})$ are undetectable within current measurement error. Incorporating a local ocean-tide model for the northeastern Pacific Ocean reduces the $\mathrm{M}_{2}$ vertical residuals by an average of $0.2 \mathrm{~mm}$, with improvements of up to $5 \mathrm{~mm}$ at some coastal stations. Estimated RMS observational uncertainties in the vertical component for the $\mathrm{M}_{2}$ and $\mathrm{O}_{1}$ tides are approximately $\pm 0.08 \mathrm{~mm}$ at the two-sigma level $( \pm 0.03 \mathrm{~mm}$ in the horizontal components), and $\pm 0.21 \mathrm{~mm}$ for the $\mathrm{M}_{\mathrm{f}}$ harmonic ( $\pm 0.07 \mathrm{~mm}$ in the horizontal components). For the $\mathrm{M}_{2}$ harmonic, discrepancies between predicted and observed OTL displacements exceed observational uncertainties by about one order of magnitude. None of the ocean tide and Earth model combinations is found to reduce the $\mathrm{M}_{2}$ residuals below the observational uncertainty, and no single forward model provides a best fit to the observed displacements across all tidal harmonics and spatial components. For the $\mathrm{O}_{1}$ harmonic, discrepancies between predicted and observed displacements are generally several-fold larger than the observational uncertainties. For the $\mathrm{M}_{\mathrm{f}}$ harmonic, the discrepancies are roughly within a factor of two of the observational uncertainties. We find that discrepancies between predicted and observed OTL displacements can be significantly reduced by removing a network-uniform tidal-harmonic displacement, and that the remaining discrepancies exhibit some regional-scale spatial coherency, particularly for the $\mathrm{M}_{2}$ harmonic. We suggest that the remaining discrepancies for the $\mathrm{M}_{2}, \mathrm{O}_{1}$ and $\mathrm{M}_{\mathrm{f}}$ tides cannot be fully explained by measurement error and instead convey information about deficiencies in ocean-tide models and deviations from spherically symmetric Earth structure.

Key words: Composition and structure of the continental crust; Composition and structure of the mantle; Structure of the Earth; Loading of the Earth; Tides and planetary waves; Planetary interiors. 


\section{INTRODUCTION}

Earth deforms in response to the redistribution of ocean water due to tidal forcing in a process known as ocean tidal loading (OTL). OTL-induced deformation can be measured using a variety of geodetic techniques, including Global Navigation Satellite Systems (GNSS), gravimeters, tiltmeters, strainmeters and very long baseline interferometers (e.g. Baker 1984; Baker et al. 1996; Ito \& Simons 2011; Yuan et al. 2013; Agnew 2015; Bos et al. 2015; Penna et al. 2015; Martens et al. 2016b). GNSS has emerged in recent years as a preferred method for investigating tidally induced Earth deformation (e.g. Agnew 2015). Advantages of GNSS include: receivers deployed around the world with a relatively high spatial density in many regions; surface deformation monitored continuously at relatively high sampling rates (typically subsecond to 30 -s intervals); direct inference of surface displacements, rather than derivatives of displacements (i.e. strain and tilt) that can be highly sensitive to local structural variations; and processing methods capable of producing precise estimates of surface displacement, typically on the order of $1 \mathrm{~mm}$ or better for each epoch (e.g. Agnew 2015; Herring et al. 2016).

OTL-induced deformation is sensitive to both the distribution and weight of the surface load as well as to the mechanical and density properties of Earth's interior (e.g. Farrell 1972a; Agnew 2015). We can therefore use precise observations of OTL to constrain allowable models for Earth structure as well as to refine models of the ocean tides. Ocean tides exhibit significantly greater spatial complexity than the solid-Earth body tides due to continental boundaries and bathymetry, which interrupt the tidally driven flow of the water (e.g. Pugh \& Woodworth 2014). The spatial complexity of the ocean load allows the structure of the Earth to be sampled at a wide variety of spatial wavelengths, including at shallower depths important to mantle convection and plate tectonics. Body tides are long-wavelength features that sample larger scale averages of Earth structure with sensitivity to deep-Earth properties (e.g. Latychev et al. 2009; Lau et al. 2017). Moreover, the broad temporal spectrum of the total ocean tide allows the deformation response of the Earth to be probed across a range of periods between characteristically seismic (seconds to hours) and glacial (thousands of years).

On average, tidal height variations in the ocean reach up to a few meters and amplitudes of surface displacements caused by OTL reach up to several centimeters in coastal areas (e.g. Lyard et al. 2006; Ito \& Simons 2011; Yuan et al. 2013; Bos et al. 2015; Martens et al. 2016b). Ocean-tide models, constrained in part by satellite measurements of sea-surface height, are now highly accurate in the deep ocean and no longer considered to be a dominant source of error in OTL analyses in most regions (e.g. Stammer et al. 2014; Bos et al. 2015; Martens et al. 2016b). The accuracy of oceantide models generally becomes degraded around complex coastlines and shallow seas, where satellite measurements are challenging and non-linear effects become important (e.g. Egbert et al. 2010; Pugh \& Woodworth 2014; Stammer et al. 2014). Furthermore, oceantide models are generally specified on regular, global grids with resolutions that do not precisely match the geometries of intricate coastlines. Large vector differences between OTL observations and predictions, particularly in coastal areas (e.g. Khan \& Tscherning 2001; King et al. 2005; Martens et al. 2016b), present opportunities to use geodetic data to further refine the ocean-tide models.

Also of interest is using geodetic observations of OTL response to constrain allowable models for Earth structure (Ito \& Simons 2011; Bos et al. 2015; Martens et al. 2016a, 2019). The theory behind load-induced Earth deformation long predates the ability to measure deformation with sufficient accuracy (e.g. Darwin 1898; Farrell 1972b; Okubo \& Saito 1983). Recent advances in satellite geodesy, however, including the proliferation of GNSS receivers worldwide, have now made structural investigations tractable. Ito \& Simons (2011) inverted residual OTL displacements in the western United States for refined elastic and density parameters through the asthenosphere, albeit using a relatively short time span of data and without accounting for load-induced geocentre motions. Bos et al. (2015) used observations of OTL response in western Europe to probe upper mantle anelasticity at semidiurnal tidal periods. Yuan et al. (2013) computed residuals between observed and predicted OTL response for a global network of GNSS receivers and found regional-scale spatial coherency, suggesting possible deficiencies in the assumed spherically symmetric and elastic Earth model. An investigation into OTL response in South America also revealed spatially coherent vector differences between OTL observations and predictions, which were generally on the order of $0.3 \mathrm{~mm}$ or less in all three spatial components of surface displacement (Martens et al. 2016b). Moreover, Martens et al. (2016b) found similarities between predictions and observations even for the small-amplitude principal lunar fortnightly tide $\left(\mathrm{M}_{\mathrm{f}}\right)$, although measurement uncertainties were larger in this frequency band than for the semidiurnal and diurnal tides. Sensitivity analyses of Earth's response to surface loading indicate peak sensitivities to structure within the crust and upper mantle (Ito \& Simons 2011; Martens et al. 2016a). As such, OTL-induced deformation provides a valuable opportunity to perform inversions for structure using geodetic data sets that are complementary to traditional seismic methods.

Here, we perform a detailed case study of ocean tidal loading in Alaska using continuous GNSS data from the Global Positioning System (GPS). We process the raw GPS data to derive time-series of surface displacements with subdaily resolution in three dimensions (east, north and up). We then perform a tidal harmonic analysis to estimate the amplitudes and phases of individual tidal harmonics, taking care to correct for harmonic modulations and non-linear effects in shallow water. We then compare the observed OTL surface displacements with forward calculations of deformation in three tidal-frequency bands: semidiurnal, diurnal and fortnightly. Since tidal patterns are similar within each frequency band, we consider only a dominant tidal harmonic from each band: the principal lunar semidiurnal harmonic, $\mathrm{M}_{2}$, with a period of $12.42 \mathrm{hr}$; the principal lunar diurnal harmonic, $\mathrm{O}_{1}$, with a period of $25.82 \mathrm{hr}$; and the principal lunar fortnightly harmonic, $\mathrm{M}_{\mathrm{f}}$, with a period of $13.66 \mathrm{~d}$. We then interpret the residual displacements in the context of GPS uncertainty estimates, modelling assumptions, ocean-tide distribution and regional Earth structure.

Ocean tidal loading has previously been explored in Alaska, albeit with a limited number of stations, short record lengths, outdated ocean-tide models, and a focus on only the semidiurnal tidal species. Khan \& Tscherning (2001) and Khan \& Scherneck (2003) investigated Earth's displacement response to semidiurnal ocean tidal loading at two GPS stations in Alaska over a period of $49 \mathrm{~d}$, and found discrepancies between observations and predictions of about $2 \mathrm{~mm}$ in the up component and $1 \mathrm{~mm}$ in the north component for the $\mathrm{M}_{2}$ harmonic. Zürn et al. (1976) considered discrepancies between the observed and modelled gravity response of the Earth to direct tidal forcing at five gravimeter stations in southern Alaska, and found that much of the residual could be explained by the effects of ocean tidal loading. We build upon and modernize the prior studies by processing data from 131 GPS receivers in the Plate Boundary Observatory (PBO) network throughout Alaska for a period of over 5 yr: 1 January 2012 to 28 February 2017. Fig. 1(a) shows the 


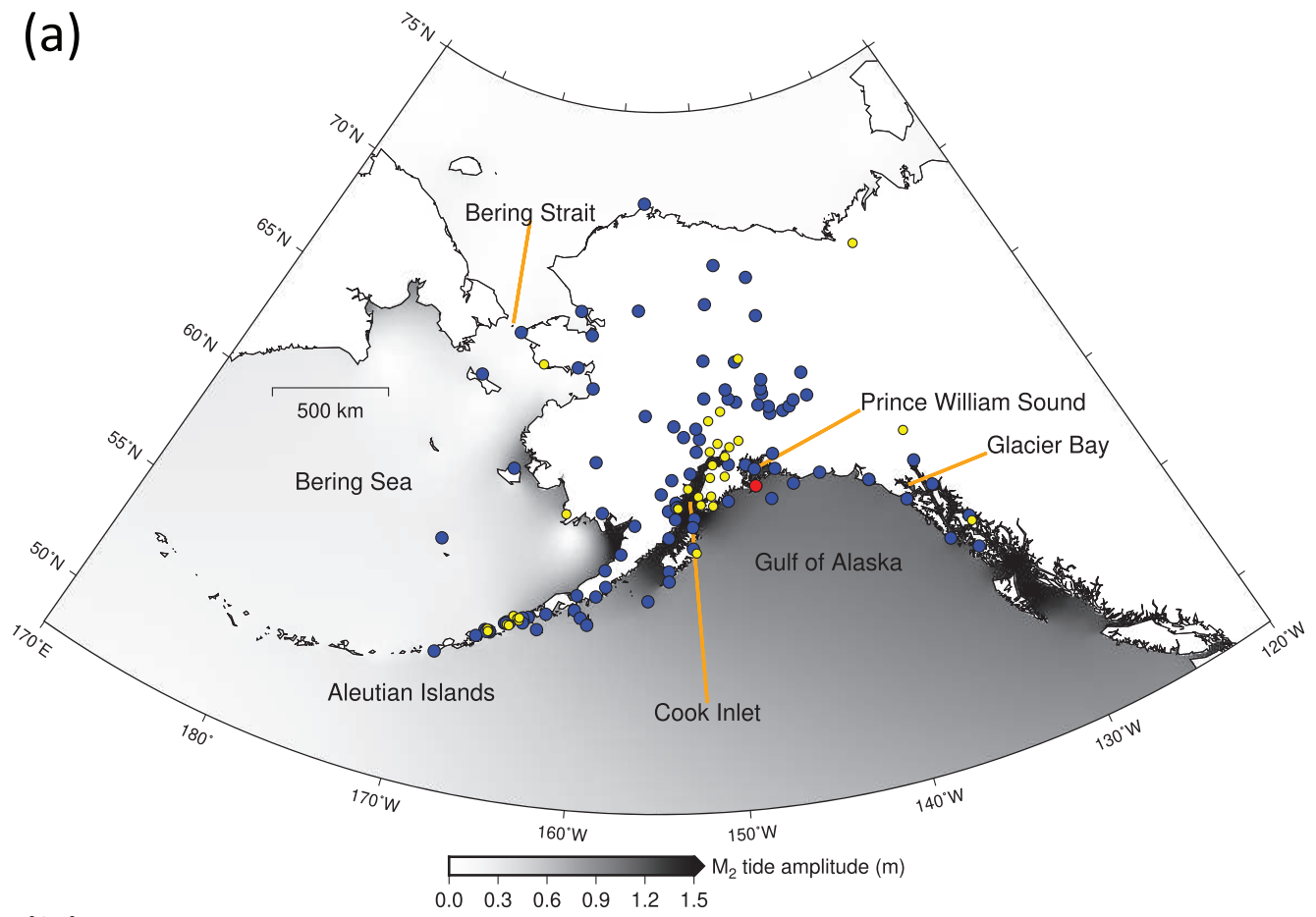

(b)

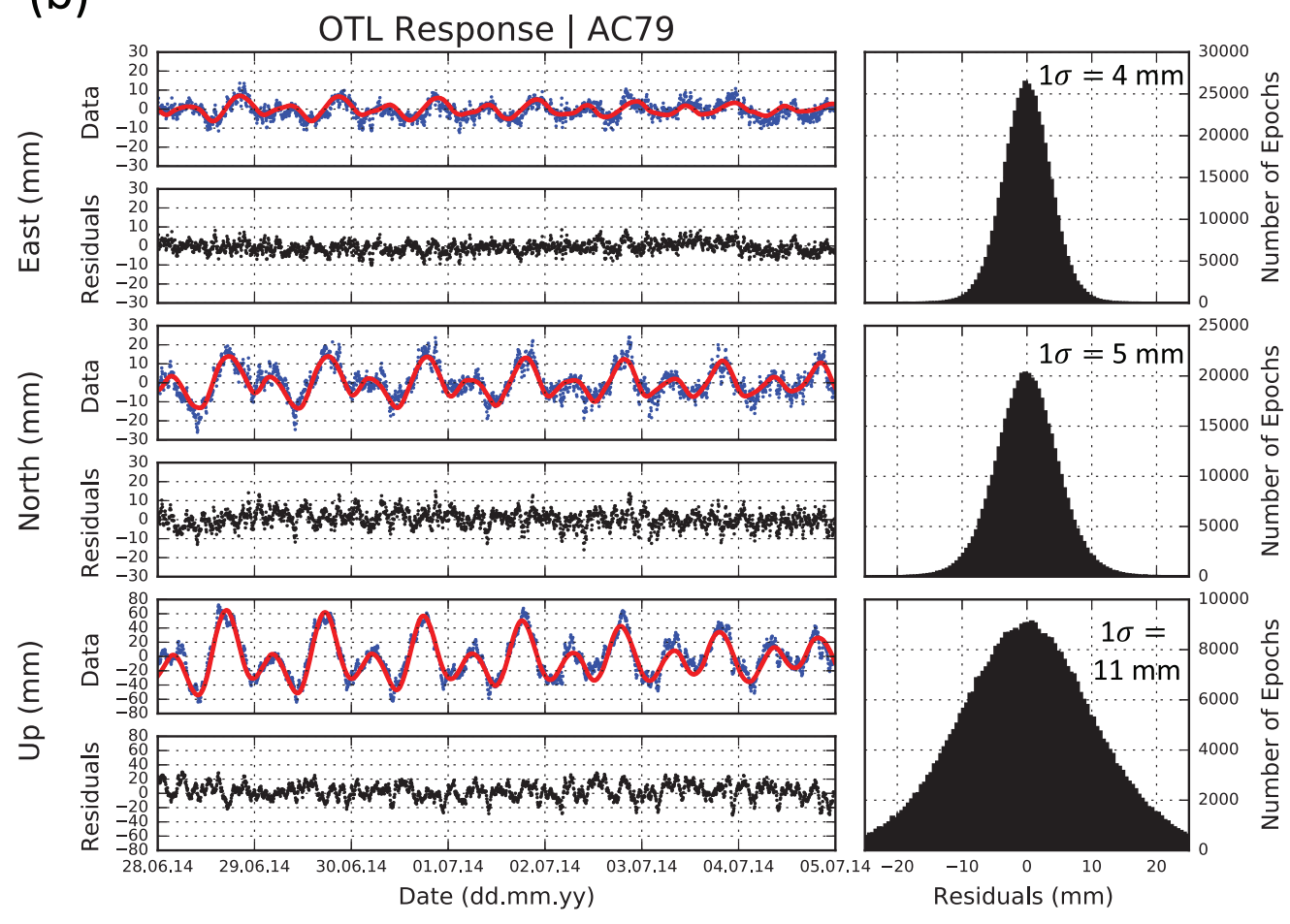

Figure 1. (a) Map of Alaska showing GPS station locations and key geographic regions. GPS stations considered in our analysis are plotted as coloured dots. The red dot denotes the station considered in panel (b). The yellow dots denote stations that are excluded from statistical and comparative analyses of predictions and observations due to anomalous signals in the time-series; predictions and observations for all stations shown in the map are provided in the Supporting Information. (b) GPS-inferred displacement time-series and tidal-harmonic model fit for PBO station AC79, which is located on Montague Island in the Gulf of Alaska. The tidal-harmonic fit has been made to the full time-series of processed data $(\approx 5 \mathrm{yr})$, but the figure shows an arbitrary snapshot of one week from summer 2014. The panels on the left side depict the GPS time-series: the upper two panels show the east component of displacement; the centre two panels show the north component of displacement; and the bottom two panels show the up component of displacement. For each pair, the top panel shows the GPS time-series for the station (blue dots) at 5-min temporal resolution with the tidal-harmonic fit to the data overlaid (red line). The bottom panel in each pair shows the residual time-series after the fitted tidal harmonics are removed (black dots). The panels on the right side depict residual displacements in the east (top panel), north (middle panel) and up (bottom panel) components for the full time-series $(\approx 5 \mathrm{yr})$. The residual value at one standard deviation from the mean is annotated on each panel. Particularly for long data records, estimates of uncertainties for individual tidal harmonics (eqs 2 and 3 ) can be much less than the standard deviation for all residuals due to the periodicity of the tides. 
Alaska network along with key geographic locations. We consider three distinct tidal species (semidiurnal, diurnal and fortnightly) as well as all three displacement components (east, north and up). Furthermore, we take advantage of contemporary, high-resolution ocean-tide models to generate improved predictions of Earth's response to OTL. We also explore a range of different ocean-tide and Earth-structure models, and evaluate the discrepancies between predictions and observations for each model pair.

\section{KINEMATIC GPS PROCESSING}

Standard GPS-processing methods involve modelling and removing both the solid Earth tides and the ocean load tides prior to estimating receiver positions, most often using a static parameter-estimation approach (e.g. Herring et al. 2016). Here, we seek to retain the ocean load tides in the GPS time-series and estimate the load-tide harmonics at the post-processing stage using harmonic-analysis techniques. We therefore developed a custom GPS-processing strategy for the specific purpose of analysing OTL-induced deformation, which closely follows the methods of Martens et al. (2016b). In particular, we process the raw RINEX-formatted GPS data using the GIPSY software (version 6.4), which uses a precise point positioning algorithm (Zumberge et al. 1997).

The 15-s data are processed in kinematic mode to estimate receiver positions at 5-min intervals. Using GIPSY, we remove the solid-Earth body and pole tides according to calculations provided by the International Earth Rotation Service (IERS, Petit \& Luzum 2010). We do not model and remove the ocean load tides. Station positions are estimated stochastically using a random-walk procedure with a coordinate process noise setting of $5.7 \times 10^{-7} \mathrm{~km} \mathrm{~s}^{-\frac{1}{2}}$, which is a GIPSY-recommended value for slow-moving platforms (software documentation) and also consistent with the synthetic tests of Martens et al. (2016b).

Nominal station positions are first obtained from the headers of RINEX files, and then updated after a preliminary run of GIPSY for each station. In order to mitigate potential inaccuracies in position estimation at the start and end of each day, we process data in $30 \mathrm{hr}$ batches (i.e. a full day plus $3 \mathrm{hr}$ on either side of the day) and extract only the central $24 \mathrm{hr}$ of position estimates. We use precise satellite orbit and clock products from the Jet Propulsion Laboratory (JPL) in final and fiducial form ('flinnR' format; version 'repro2.1'). We adopt a minimum elevation cutoff of $7^{\circ}$ above the horizon, below which satellite signals are discarded by the position-estimation algorithm. Satellite signals are weighted with the square root of the sine of the elevation. We also apply phase-centre corrections to the receiver antennas, extrapolating the models down to $7^{\circ}$-elevation as needed. GIPSY performs single-receiver integer-phase ambiguity resolution using pre-computed wide-lane and phase-bias estimates from a global network of reference receivers (Bertiger et al. 2010).

To limit the unintentional absorption of tidal signal into the estimate of tropospheric delays, or vice versa, we estimate the tropospheric delays stochastically and simultaneously with the coordinate positions relative to nominal values at high temporal resolution (Penna et al. 2015; Martens et al. 2016b). Specifically, we estimate signal-propagation delays through the troposphere using numerical weather models in combination with stochastic modelling. Nominal values for the wet and dry tropospheric zenith delay are extracted from the Vienna Mapping Function (VMF1) grids, which are based on numerical weather models from the European Centre for Medium Range Weather Forecasts (ECMWF) with 6-hr temporal resolution (Boehm et al. 2006). Zenith delays are mapped to lower elevations using the VMF1 mapping functions. We then estimate the tropospheric zenith delay stochastically, simultaneously with the coordinate positions, relative to the nominal delay values using a process noise setting of $5.0 \times 10^{-8} \mathrm{~km} \mathrm{~s}^{-\frac{1}{2}}$ (cf. Martens et al. 2016b). Horizontal gradients in the tropospheric wet delay are also estimated stochastically using a process noise setting of $5.0 \times 10^{-9} \mathrm{~km} \mathrm{~s}^{-\frac{1}{2}}$ (Bar-Sever et al. 1998). Furthermore, we apply 2nd-order corrections to the signal-propagation delay through the ionosphere with an effective shell height of $600 \mathrm{~km}$.

\section{TIDAL HARMONIC ANALYSIS}

We use the analysis methods described in Martens et al. (2016b) to estimate the tidal harmonics in three dimensions (east, north and up). We model the total tidal displacement for each spatial component as a function of time, $P(t)$, as a sum of sinusoids:

$$
\begin{aligned}
P(t)= & \sum_{n=1}^{N} c_{n} f_{n}(t) \cos \left(V_{n}(t)+u_{n}(t)\right) \\
& +s_{n} f_{n}(t) \sin \left(V_{n}(t)+u_{n}(t)\right),
\end{aligned}
$$

where $c_{n}$ and $s_{n}$ are the in-phase and quadrature amplitudes of tidal harmonic $n, V_{n}$ is the astronomical argument, $N$ is the total number of harmonics considered, $t$ is time and $f_{n}$ and $u_{n}$ are the nodal factors needed to express modulation of the tidal harmonic (Foreman et al. 2009; Martens et al. 2016b). We evaluate $V, f$ and $u$ at each epoch in the time-series. The amplitudes and phases are given by $A_{n}=\sqrt{c_{n}^{2}+s_{n}^{2}}$ and $\phi_{n}=\operatorname{atan} 2\left(s_{n}, c_{n}\right)$, respectively.

For the selection of primary harmonics to include in the inversion, we consider the Rayleigh frequency-resolution criterion with a cut-off value of 1.1 cycles for the Rayleigh parameter (e.g. Godin 1972; Foreman 1977). Harmonics with the largest equilibriumtide amplitudes are prioritized over smaller-amplitude harmonics within the same frequency band. We base the amplitude rankings on the Cartwright-Taylor-Edden (CTE) equilibrium tide catalogue (Cartwright \& Taylor 1971; Cartwright \& Edden 1973).

In shallow seas and estuaries, non-linear effects can become important due to the increasing influence of friction between the water and the sea floor (e.g. Pugh \& Woodworth 2014). We account for contributions to the time-series by a selection of shallow-water harmonics that arise from distortions and interactions of the semidiurnal and diurnal astronomical tides. A list of the nonlinear harmonics considered in our study is provided with the Supporting Information of Martens et al. (2016b).

Prior to fitting tidal harmonics to the time-series, we first remove offsets associated with known earthquakes and equipment changes using a catalogue of events and offset amplitudes provided by the UNAVCO consortium (Herring et al. 2016). We also account for long-term plate motions and regional-scale glacial isostatic adjustment by estimating and removing a linear trend from each timeseries. In addition, we discard isolated segments of data that are fewer than $60 \mathrm{~d}$ in length and separated from data on both sides by at least $60 \mathrm{~d}$, since isolated segments of data can sometimes be associated with instrument malfunction. Moreover, we flag and remove the most egregious outliers from each time-series using a median absolute deviation technique with a conservative cutoff of 15 standard deviations. Fewer than 0.1 per cent of the data are flagged as outliers for more than 75 per cent of the stations.

The harmonic analysis that we perform is relatively robust to anomalous data, particularly given long time windows of data and the use of an iterative reweighted least-squares (IRLS) regression 
algorithm that converges to an L1-norm solution (e.g. Aster et al. 2013; Martens et al. 2016b). Thus, we can generally estimate tidal parameters with reasonable precision even for stations with poorer data quality or additional un- and mismodelled signals. Nevertheless, we discarded several stations from the analysis due to identifiable and explicable unwanted signals that could potentially bias the estimates of the tides. Specifically, we exclude AB11, AB12, AB53, AC32, AC33, AC47, AV04, AV20, AV25, AV27, AV29 and AV35 on the basis of abnormal seasonal spikes in the displacement time-series that are likely caused by the build-up of snow and ice on the GPS receivers. Stations with minor or short-term anomalies are retained. We also exclude AV12, AV14, AV36, AV37 and AV39 as well as AC03, AC06, AC11, AC15, AC23, AC35, AC36, AC44, AC53, AC75, ATW2 and SELD due to the presence of long-period non-linear signals in the time-series that likely reflect transient volcanic and tectonic deformation, respectively (e.g. Fu \& Freymueller 2013). Long-period (e.g. weekly to multiyear) transients have practically no effect on estimates of short-period tides due to the large separation in frequency, but may influence estimates of long-period tides. In total, we are left with 102 stations after the exclusions. Although we exclude the aforementioned stations from further statistical analysis, we provide the complete set of tidal observations and predictions for all stations in the Supporting Information.

To quantify uncertainties in tidal parameters estimated from the GPS data, we perform a spectral analysis of each residual time-series (i.e. with all fitted harmonics removed) and estimate the noise within a frequency window of half-width 0.5 cycles per month centred on each tidal band (Pawlowicz et al. 2002; Martens et al. 2016b). Power spectral densities are calculated using multitaper methods from the NiTime Python library, implemented with adaptive weighting of tapers and a default bandwidth of four times the fundamental frequency of each time-series (Thomson 2007; NiTime Developers 2020). The average of the noise power spectrum around each tidal harmonic provides an estimate of the variance in the harmonic coefficients ( $c_{n}$ and $s_{n}$ from eq. 1$)$. We propagate the errors to map the variance of the harmonic coefficients into the variance of amplitude and phase:

$$
\begin{aligned}
\sigma_{A_{n}} & =\sqrt{\left(\frac{\partial A_{n}}{\partial c_{n}}\right)^{2} \sigma_{c_{n}}^{2}+\left(\frac{\partial A_{n}}{\partial s_{n}}\right)^{2} \sigma_{s_{n}}^{2}} \\
& =\frac{1}{A_{n}} \sqrt{c_{n}^{2} \sigma_{c_{n}}^{2}+s_{n}^{2} \sigma_{s_{n}}^{2}} \\
\sigma_{\phi_{n}} & =\sqrt{\left(\frac{\partial \phi_{n}}{\partial c_{n}}\right)^{2} \sigma_{c_{n}}^{2}+\left(\frac{\partial \phi_{n}}{\partial s_{n}}\right)^{2} \sigma_{s_{n}}^{2}} \\
& =\frac{1}{A_{n}^{2}} \sqrt{s_{n}^{2} \sigma_{c_{n}}^{2}+c_{n}^{2} \sigma_{s_{n}}^{2}},
\end{aligned}
$$

where $\sigma_{A_{n}}$ represents the standard deviation in the amplitude, $\sigma_{\phi_{n}}$ represents the standard deviation in the phase and $\sigma_{c_{n}}$ and $\sigma_{s_{n}}$ represent the standard deviations in the harmonic coefficients (assumed equivalent for a given tidal harmonic $n$ ).

Fig. 1(b) shows an example of the tidal-harmonic fits to threecomponent displacement time-series from station AC79 on Montague Island in the Gulf of Alaska. We performed the tidal-harmonic inversions using the full 5.16-yr span of data considered in our analysis. Station AC79 includes several data gaps; the lengths and times of data gaps for all stations, which do not affect the inversion other than to reduce the amount of available data, are provided in the Supporting Information (Figs S10-S13). To visualize the tidal harmonics clearly, which are dominated by energy at semidiurnal and diurnal periods, we show only an arbitrarily selected one-week snapshot of the total tidal fit in Fig. 1(b). The histograms in Fig. 1(b) depict the distributions of residuals from the entire time span of data for AC79.

\section{FORWARD MODELLING OF ELASTIC OTL RESPONSE}

We model the 3-D (east, north and up) surface displacements caused by mass loading from ocean tides using the LoadDef software (Martens et al. 2019). LoadDef requires only an ocean-tide model and a radial profile of Earth structure to calculate the surface displacements induced by the load tide. The Earth model is assumed to be spherically symmetric, non-rotating, elastic, isotropic (SNREI), self-gravitating and hydrostatically pre-stressed. Load Love numbers are computed by integrating the equations of motion for spheroidal deformation through the interior layers of the Earth (e.g. Alterman et al. 1959; Longman 1962, 1963; Takeuchi \& Saito 1972; Farrell 1972a; Martens et al. 2016a). Boundary conditions appropriate for mass loading (i.e. a normal stress and a gravitational body force) are applied at the surface (e.g. Farrell 1972a; Okubo \& Saito 1983; Guo et al. 2004; Martens et al. 2016a).

Load Love numbers are then combined in spherical-harmonic expansions to compute load Green's functions (LGFs) for vertical and horizontal displacement, which characterize the deformation response of the SNREI Earth to a point load of unit mass (e.g. Farrell 1972a; Martens et al. 2016a). Beyond spherical-harmonic degree 10000 , we assume asymptotic values for the load Love numbers. To speed convergence, we apply a second-order Kummer's transformation to the infinite series as well as a disk factor of radius $0.1^{\circ}$ beyond an angular distance of $10^{\circ}$ from the load point (e.g. Martens et al. 2016a). We transform the degree-1 load Love numbers into the CM reference frame prior to computation of the LGFs (Blewitt 2003; Fu et al. 2012; Martens et al. 2016b). The LGFs only vary as a function of angular distance from the load point; since spherical symmetry of the Earth has been assumed and the applied force is vertical, the LGFs are symmetric about the load point with respect to azimuth.

To model displacements caused by the full distribution of the tidal load, we convolve the LGFs with models of the ocean tides. Convolutions are made separately for the real and imaginary components of ocean-tide height; surface displacements are converted back to amplitude and phase at the post-processing stage. We assume a globally uniform value for sea water density of $1035 \mathrm{~kg} \mathrm{~m}^{-3}$. LoadDef creates a template grid over Earth's surface, centred on the location of the observation point, to perform the discrete convolution. Surface displacements are most sensitive to colocated and nearby loads; thus, we generate the template grid with highest resolution in the immediate vicinity of the station ( $\approx 10 \mathrm{~m}$ within $2 \mathrm{~km}$ of the station) and gradually decrease the resolution with greater distance from the station (to $\approx 50 \mathrm{~km}$ beyond $90^{\circ}$ angular distance). The ocean-tide heights are interpolated onto the template grid, and multiplied by the integrated LGF and load density associated with each grid cell. Within each grid cell, the LGF is assumed to be constant. To ensure that cells over land do not contribute to the ocean load, we apply a land-sea mask based on ETOPO1 (Amante \& Eakins 2009) and the SCAR Antarctic Digital Database (https: //www . add.scar.org).

The assumed grid resolution for the discrete convolution as well as the uniform value adopted for sea water density can affect the 
predicted OTL displacements. Increasing the resolution of the template grid for the discrete convolution by a factor of two has only a small effect on the predicted OTL displacements: median differences of $0.01 \mathrm{~mm}$ in amplitude for the $\mathrm{M}_{2}$ tide (or about 0.1 per cent) and an even smaller effect on the $\mathrm{O}_{1}$ and $\mathrm{M}_{\mathrm{f}}$ tides (less than a few microns). We also tested two separate values for the density of sea water: 1035 and $1030 \mathrm{~kg} \mathrm{~m}^{-3}$. Median vector differences between predicted OTL displacements for the $\mathrm{M}_{2}$ harmonic computed with the two different values for sea water density are $0.02 \mathrm{~mm}$ in the horizontal components and $0.06 \mathrm{~mm}$ in the up component, which are about one order of magnitude smaller than differences between predicted OTL displacements computed from different Earth and tide models. For the $\mathrm{O}_{1}$ and $\mathrm{M}_{\mathrm{f}}$ tides, the median differences are even smaller (see Supporting Information).

\section{PREDICTED AND OBSERVED OCEAN TIDAL LOADING}

Fig. 2 shows OTL displacements, both observed by GPS (panel a) and predicted by LoadDef (panel b), for the $\mathrm{M}_{2}$ harmonic. We portray the load-tide displacements using particle motion ellipses (PMEs, e.g. Ito \& Simons 2011; Martens et al. 2016a,b, 2019). Since the period of the $\mathrm{M}_{2}$ tidal forcing and response (12.42 hr) is the same in all three spatial components, the PMEs are closed in 3-D space. The largest $\mathrm{M}_{2}$ load-tide displacements, which exceed $3 \mathrm{~cm}$ in vertical amplitude, are observed along the coastlines adjacent to the Gulf of Alaska where the $\mathrm{M}_{2}$ ocean-tide amplitudes are relatively large. $\mathrm{M}_{2}$ OTL displacements diminish to less than $1 \mathrm{~cm}$ in northern Alaska because the $\mathrm{M}_{2}$ ocean-tide amplitudes are smaller in that region. The semimajor axis of each PME is aligned towards the region of maximum tidal loading and unloading.

Panel (c) of Fig. 2 displays the estimated uncertainties in the observed load-tide displacement amplitudes, which are on the order of a fraction of a millimeter in all three spatial components (quadratic means of $\pm 0.03 \mathrm{~mm}$ in the horizontal components and $\pm 0.07 \mathrm{~mm}$ in the vertical component); phase-uncertainty information is not shown in the figure. Vector differences between the predicted and observed displacements are shown in panel (d) of Fig. 2. The residual surface displacements exceed the observational uncertainties by more than one order of magnitude, indicating that the tidal deformations are well measured by the GPS and that the residuals convey information about deficiencies in the forward model.

Figs 3 and 4 show observed and predicted OTL displacements and estimated uncertainties for the $\mathrm{O}_{1}$ and $\mathrm{M}_{\mathrm{f}}$ ocean-load tides, respectively. The residual displacements for the $\mathrm{O}_{1}$ harmonic, which are sub-mm in scale, are several-fold larger than the two-sigma observational uncertainties at most stations (quadratic means of $\pm 0.03 \mathrm{~mm}$ in the horizontal components and $\pm 0.08 \mathrm{~mm}$ in the vertical component). For the $\mathrm{M}_{\mathrm{f}}$ harmonic, the sub-mm residuals are marginally larger than the two-sigma observational uncertainties (quadratic means of $\pm 0.07 \mathrm{~mm}$ in the horizontal components and $\pm 0.21 \mathrm{~mm}$ in the vertical component). Interpretations of spatial variability for the $\mathrm{O}_{1}$ and $\mathrm{M}_{\mathrm{f}}$ residuals are therefore more tenuous than for the $\mathrm{M}_{2}$ harmonic, but are nevertheless practicable. Tables with complete amplitude and phase information for the observed OTL displacements and estimated uncertainties at all stations are provided in the Supporting Information.

Overall, observed OTL displacements inferred from GPS data bear strong $\left(\mathrm{M}_{2}\right.$ and $\left.\mathrm{O}_{1}\right)$ or moderate $\left(\mathrm{M}_{\mathrm{f}}\right)$ resemblance to the predicted OTL displacements (Figs 2-4a and b). Although the correlations are weaker for the $\mathrm{M}_{\mathrm{f}}$ harmonic due to smaller tide amplitudes and larger observational uncertainties, the observed and predicted $\mathrm{M}_{\mathrm{f}}$ PMEs also exhibit marked consistencies: the semimajor axes of both the observed and predicted PMEs are aligned predominantly in the north-south direction and the largest vertical displacements are found along the Aleutian Island chain and diminish toward the east.

\subsection{Effects of solid Earth structure and mantle anelasticity}

For the comparisons in Figs 2-4, we predict OTL displacements using an oceanless variant of the elastic Preliminary Reference Earth Model (PREM, Dziewonski \& Anderson 1981) and the FES2014b ocean-tide model (Lyard et al. 2006; Carrère et al. 2012). We create the oceanless variant of PREM by averaging the ocean layer and outermost crustal layer to form a single top layer that conserves total mass and has elastic properties equal to the original outermost crustal layer (Guo et al. 2004; Martens et al. 2019).

Next, we compare observed OTL displacements with predictions made using other models for the structure of the Earth. In particular, we consider two additional standard Earth models: STW105 (Kustowski et al. 2008) and ak135f (Kennett et al. 1995; Montagner \& Kennett 1996). We create oceanless variants of STW105 and ak135f using the same methods described above for PREM. Moreover, we consider a model for lithospheric structure specific to the Anchorage area, which we extract from LITHO1.0 at $61.2181^{\circ} \mathrm{N}, 149.9003^{\circ} \mathrm{W}$ (Pasyanos et al. 2014). Below about $60 \mathrm{~km}$ depth, we supplement the LITHO1.0 model with ak135f. Finally, we consider the effects of anelastic dispersion in the asthenosphere at tidal periods (e.g. Dahlen \& Tromp 1998; Bos et al. 2015; Wang et al. 2020), which reduces the shear modulus in the asthenosphere by about $8.5,9.1$ and 11.1 percent at the periods of the $\mathrm{M}_{2}, \mathrm{O}_{1}$ and $\mathrm{M}_{\mathrm{f}}$ harmonics, respectively. We consider only the real part of the perturbation to the shear and bulk moduli due to anelastic dispersion. Details of the Earth models used in our study are provided in the Supporting Information (Fig. S1 and Dataset 3).

Fig. 5 shows empirical cumulative distribution functions (ECDFs) of the residuals between predicted and observed OTL displacements, with different models for Earth structure assumed. Of the three harmonics we consider, the residuals are largest for $\mathrm{M}_{2}$, due primarily to its relatively large amplitude. In the $\mathrm{M}_{2}$ up component, the regionally appropriate LITHO1.0 model provides the best overall fit to the observed data, with STW105 and anelastic PREM yielding relatively small misfits as well. The LITHO1.0 and STW105 models, however, yield relatively poor fits to the observed data in the horizontal components. For the $\mathrm{M}_{2}$ tide overall, anelastic PREM yields the smallest residuals across all three spatial components. Assuming anelastic PREM and FES2014b, median residuals between predicted and observed displacements are 0.34, 0.27 and $0.55 \mathrm{~mm}$ in the east, north and up components, respectively.

For the $\mathrm{O}_{1}$ tide, anelastic PREM provides a relatively good fit to the observed data in the north and up components, but a relatively poor fit in the east component. No single Earth model performs consistently well in all three spatial components for the $\mathrm{O}_{1}$ harmonic. For the $\mathrm{M}_{\mathrm{f}}$ tide, the choice of Earth model is not significant: the ECDF curves are effectively identical. Differences in the Earth models considered here affect the predicted $\mathrm{M}_{\mathrm{f}}$ load-tide displacements at the level of tens of microns or less, which is approximately an order of magnitude smaller than present-day GPS precision.

Residuals normalized by the amplitudes of the observed OTL response at each station are shown in the Supporting Information. 

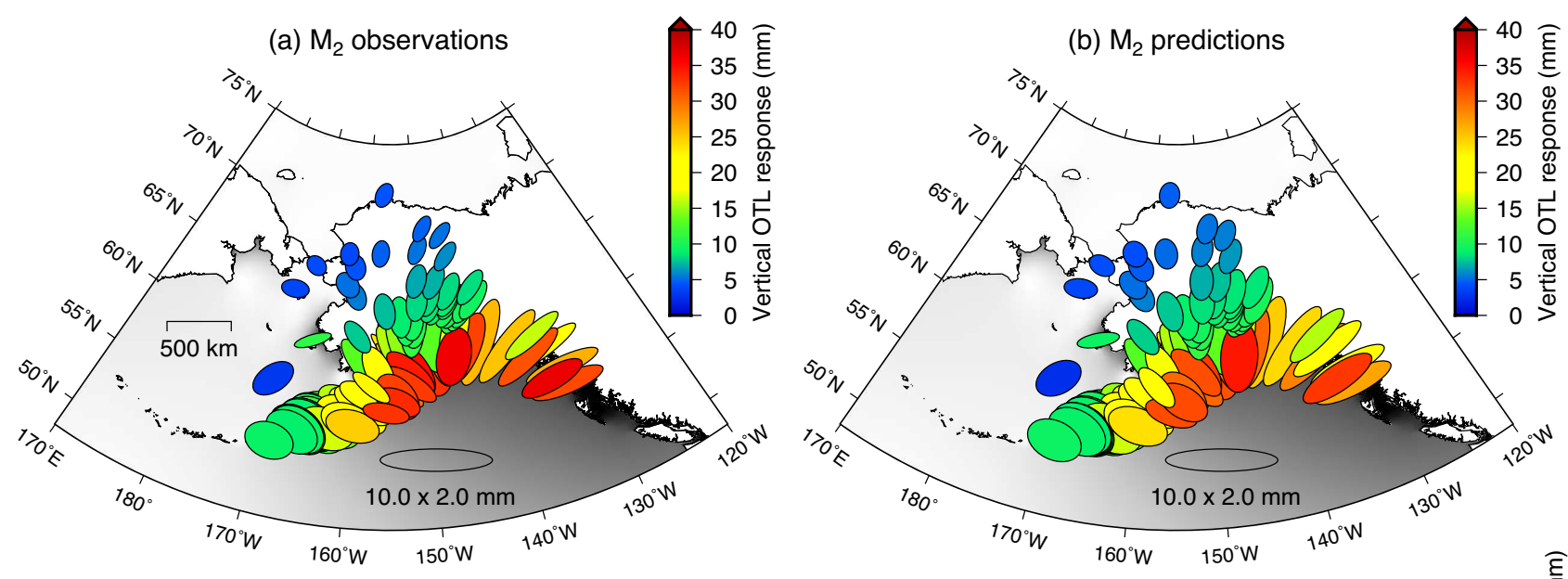

(c) $M_{2}$ uncertainties (95\% confidence)

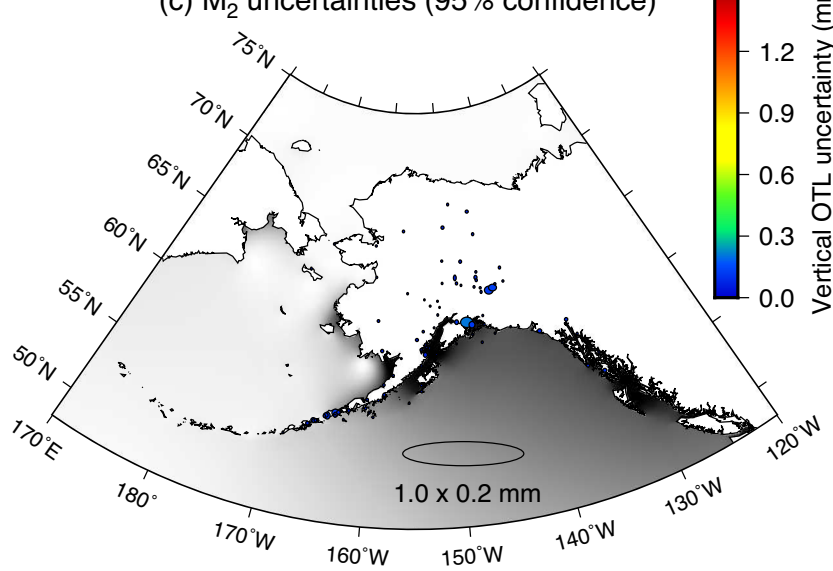

(d) $M_{2}$ residuals

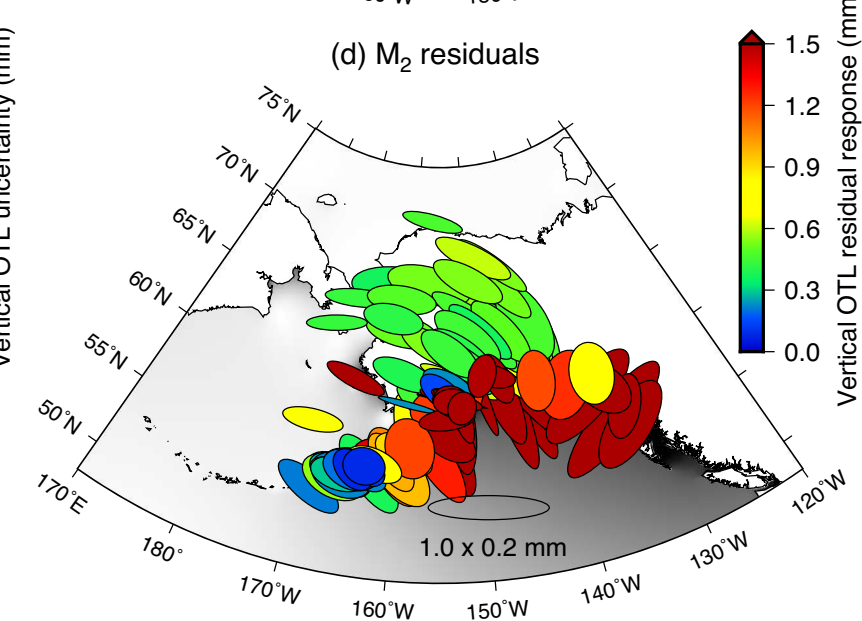

$\mathrm{M}_{2}$ tide amplitude $(\mathrm{m})$

$\begin{array}{llllll}0.0 & 0.3 & 0.6 & 0.9 & 1.2 & 1.5\end{array}$

Figure 2. (a) Observed and (b) predicted surface displacements at PBO GPS stations in Alaska caused by mass loading from the $\mathrm{M}_{2}$ ocean tide. The predictions are computed by assuming an oceanless variant of PREM and the FES2014b ocean-tide model in the CM reference frame. Panels (c) and (d) depict the two-sigma observational uncertainties and the vector differences between the predicted and observed deformation, respectively. We depict the deformation as PMEs: the sizes, shapes, and orientations of the PMEs denote horizontal displacement and the colours depict vertical displacement. Reference PMEs are shown at the bottom of each panel; note that the horizontal scales for the reference PMEs vary from panel to panel. The colour scales depicting vertical deformation also change from panel to panel. Observations and predictions are depicted at the same scale for direct comparison (panels a and b); uncertainties and residuals are depicted at the same scale for direct comparison (panels $\mathrm{c}$ and d). The observational uncertainties (panel c) are significantly smaller than the $\mathrm{M}_{2}$ OTL residuals (panel d). The uncertainties do not include phase information; the east- and north-amplitude uncertainties define the size of the ellipse and the vertical-amplitude uncertainty defines the colour.

The normalized residuals for the $\mathrm{M}_{\mathrm{f}}$ tide are several-fold larger than for the $\mathrm{M}_{2}$ and $\mathrm{O}_{1}$ tides due to relatively large observational uncertainties at the $\mathrm{M}_{\mathrm{f}}$ period and relatively small $\mathrm{M}_{\mathrm{f}}$ tidal amplitudes. Furthermore, the normalized residuals for the $\mathrm{M}_{\mathrm{f}}$ tide are approximately twice as large in the east component as in the north and up components. Even though the east component accounts for the smallest unnormalized residuals (Fig. 5), the east component of the $\mathrm{M}_{\mathrm{f}}$ OTL response has the smallest amplitude. The long-period $\mathrm{M}_{\mathrm{f}}$ tide primarily flows between polar and equatorial regions with a zonal pattern, and produces only small amounts of east-west displacement (Fig. S5 in the Supporting Information).

Comparisons between pairs of predicted OTL displacements in map view are shown in the Supporting Information. The largest discrepancies in predicted displacements are found in coastal regions around the Gulf of Alaska, where the ocean-tide heights are large and load-to-station distances are short. For the $\mathrm{O}_{1}$ harmonic, the pattern of residual OTL displacements when comparing Earth structure appears remarkably similar to the pattern for the $\mathrm{M}_{2}$ harmonic, which relates to the similar distributions of the two ocean tides in the region (Figs S2 and S3). When considering two sets of residuals between OTL predictions computed from the same pair of Earth models, the spatial discrepancies between the residuals for different tidal harmonics stem only from the load distribution. The amplitudes of the residuals, however, are smaller for the $\mathrm{O}_{1}$ harmonic than for the $\mathrm{M}_{2}$ harmonic due to smaller ocean-tide heights.

\subsection{Effects of discrepancies between ocean-tide models}

Markedly large discrepancies between OTL predictions and observations for the $\mathrm{M}_{2}$ harmonic are found around Glacier Bay in southeastern Alaska as well as in Cook Inlet and Prince William Sound near the city of Anchorage (Figs 1a and 2d). The residuals reflect both observational and modelling errors; however, since the observational errors are not abnormally large for the Glacier 

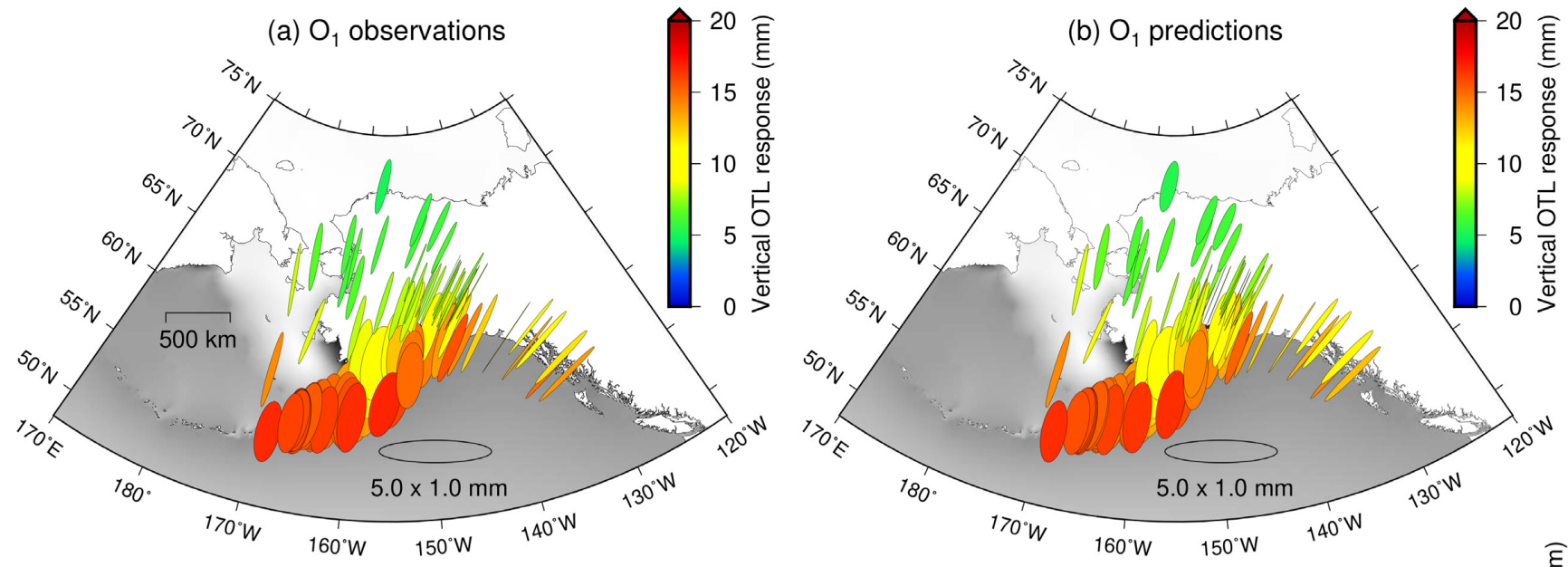

(c) $\mathrm{O}_{1}$ uncertainties (95\% confidence)

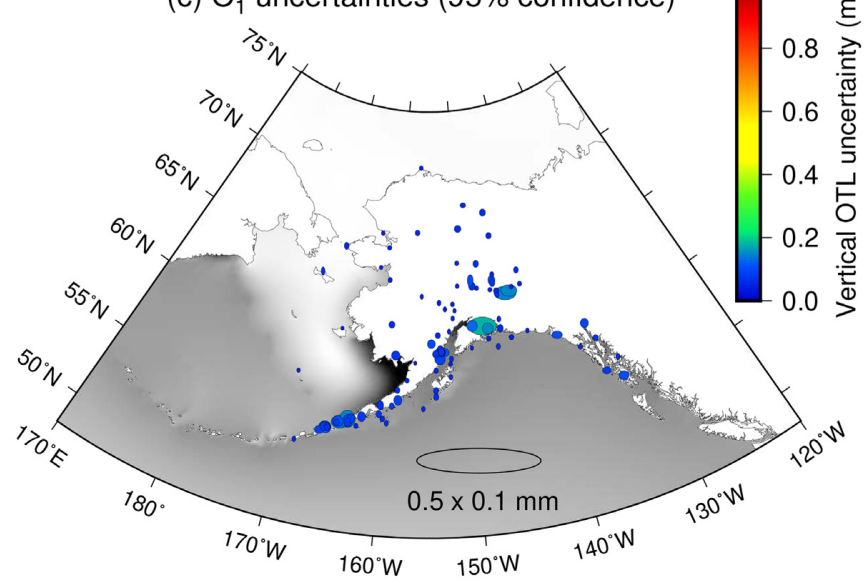

(d) $\mathrm{O}_{1}$ residuals

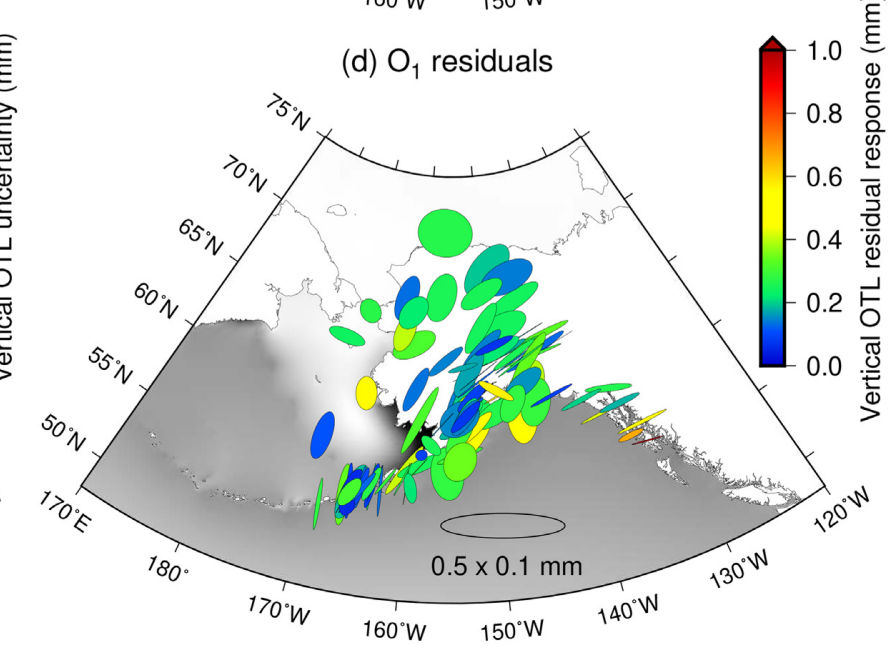

$\mathrm{O}_{1}$ tide amplitude $(\mathrm{m})$

$\begin{array}{llllll}0.0 & 0.1 & 0.2 & 0.3 & 0.4 & 0.5\end{array}$

Figure 3. Same as Fig. 2, but for the $\mathrm{O}_{1}$ tidal harmonic. Note that the reference PMEs and colour bars are scaled differently relative to Fig. 2 because the $\mathrm{O}_{1}$ tide has smaller amplitudes overall than the $\mathrm{M}_{2}$ tide (see also the ocean-tide amplitude scale at the base of each figure).

Bay, Cook Inlet or Prince William Sound GPS stations (Fig. 2), we hypothesize that most of the error stems from forward-modelling assumptions. A likely explanation for the larger-than-average discrepancies in each region is the precision of the ocean-tide model, since ocean-tide models are notoriously difficult to constrain in regions of shallow water and complex coastlines (Inazu et al. 2009; Sato 2010; Stammer et al. 2014).

To investigate the effects of tide-model discrepancies on predictions of OTL, we consider a representative sampling of modern global tide models: FES2014b (Lyard et al. 2006; Carrère et al. 2012), TPXO9-Atlas (Egbert \& Erofeeva 2002; Egbert et al. 2010), EOT11a (Savcenko \& Bosch 2012) and GOT4.10c (Ray 1999, 2013). EOT11a and GOT4.10c are empirical tide models constrained primarily by satellite altimetry data, whereas FES2014b and TPOX9-Atlas are derived from hydrodynamic models that assimilate empirical data. Since TPXO9-Atlas does not include the $\mathrm{M}_{\mathrm{f}}$ harmonic (at the time of our analysis), we use the $\mathrm{M}_{\mathrm{f}}$ harmonic from a previous version of the TPXO suite of models: TPXO8-Atlas. We also consider a regional hydrodynamic tide model specific to the Eastern North Pacific Ocean from the ADCIRC tidal database: ENPAC15 (Szpilka et al. 2018). Outside the bounds of the regional ENPAC15 model, we assume tide amplitudes and phases according to FES2014b (see the Supporting Information).
Fig. 6 shows ECDFs of the residuals between predicted and observed OTL displacements, assuming different ocean-tide models. For the up component of the $\mathrm{M}_{2}$ tide, the regional ENPAC15 model yields the best fit to the observations at most stations. In particular, the ENPAC15 model reduces some of the largest residuals that are found in coastal areas around the Gulf of Alaska and Glacier Bay by up to $5 \mathrm{~mm}$ at some stations (e.g. station AB51). The GOT4.10c model, which has the lowest spatial resolution of all tide models considered here, performs relatively poorly at coastal stations, but relatively well at inland sites. Vector differences between predicted $\mathrm{M}_{2}$ up displacements computed using different ocean tide models (e.g. ENPAC15 and GOT4.10c) can exceed $6 \mathrm{~mm}$ at some coastal stations (e.g. station LEV6), but are generally less than $1.5 \mathrm{~mm}$ at inland stations. Coastal and shelf regions commonly exhibit variability between ocean-tide models because of challenges in making satellite altimetry measurements near to the shore, non-linear effects in shallow water, and model resolution around complex coastlines (e.g. Stammer et al. 2014). Furthermore, OTL displacements are most sensitive to near-field loads (e.g. Farrell 1972b).

For the horizontal components of the $\mathrm{M}_{2}$ tide, the TPXO9-Atlas model provides the best overall fit to the observed OTL displacements. For the $\mathrm{O}_{1}$ tide, the FES2014b model outperforms the other tide models in the north and up components. TPXO9-Atlas yields 

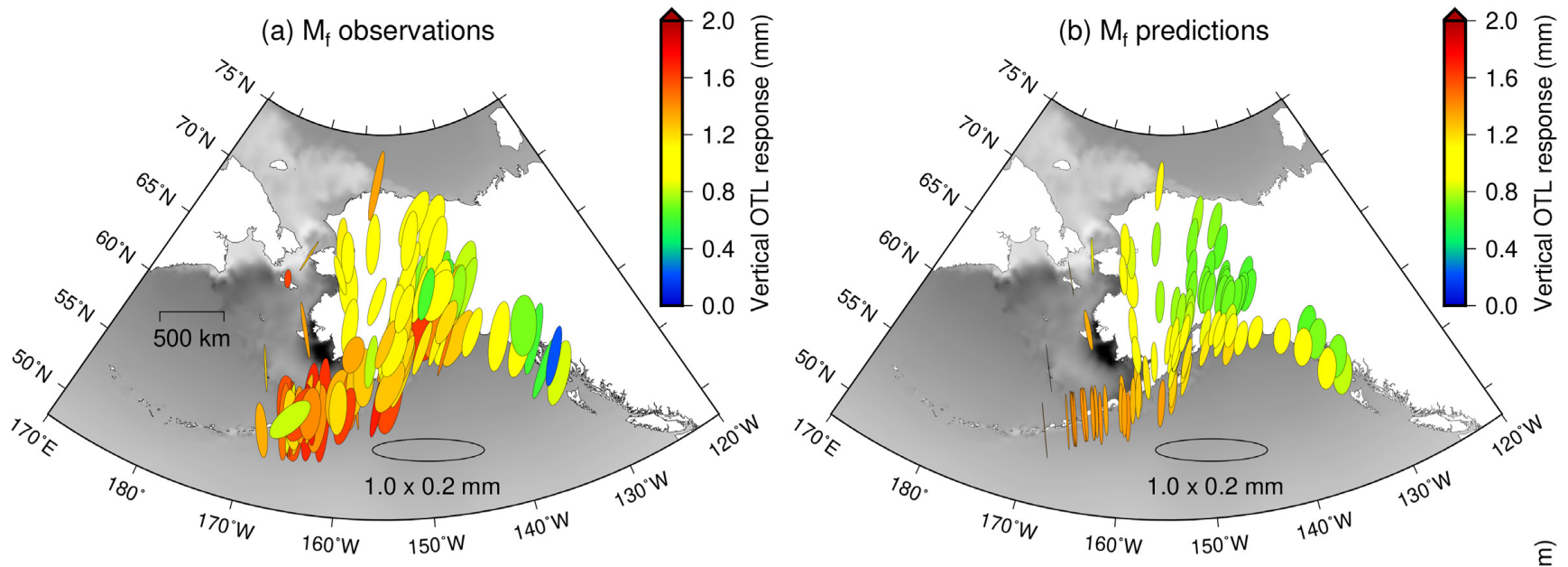

(c) $M_{f}$ uncertainties (95\% confidence)
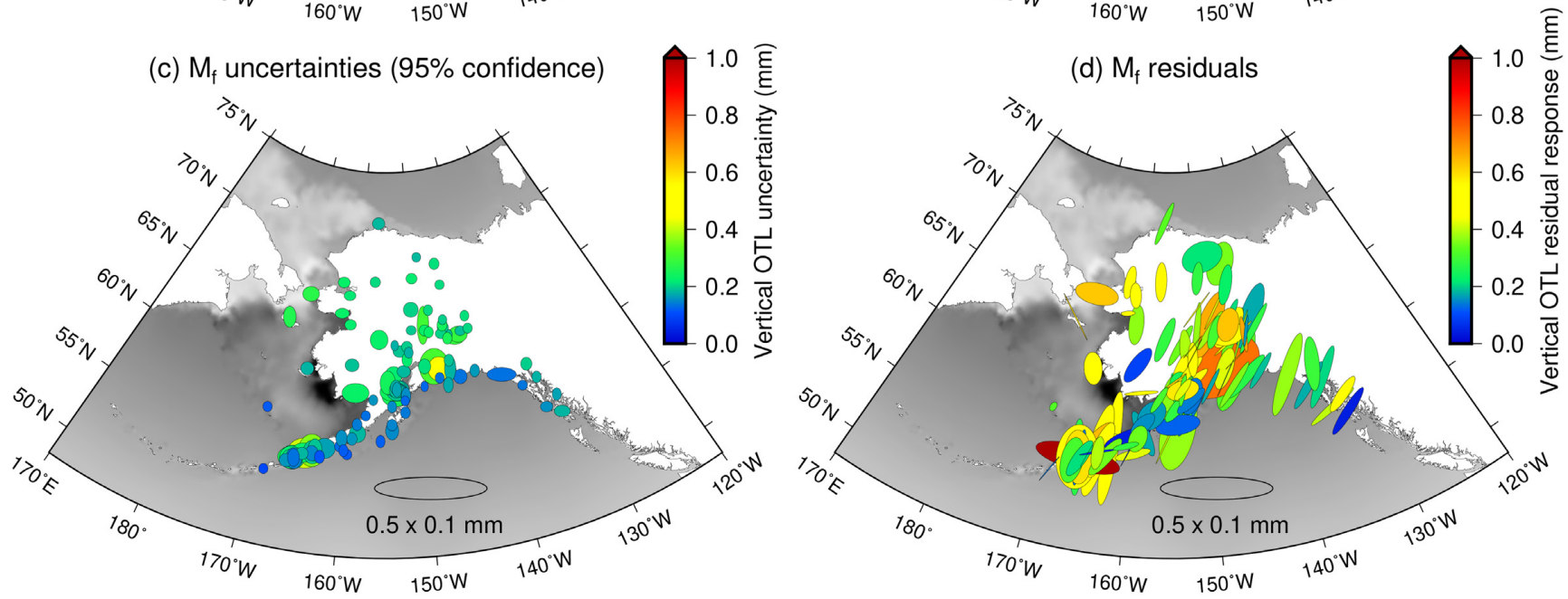

$\mathrm{M}_{\mathrm{f}}$ tide amplitude $(\mathrm{m})$

$\begin{array}{lllll}0.00 & 0.01 & 0.02 & 0.03 & 0.04\end{array}$

Figure 4. Same as Fig. 2, but for the $\mathrm{M}_{\mathrm{f}}$ tidal harmonic. Note that the reference PMEs and colour bars are scaled differently relative to Fig. 2 because the $\mathrm{M}_{\mathrm{f}}$ tide has significantly smaller amplitudes than the $\mathrm{M}_{2}$ tide (see also the ocean-tide amplitude scale at the base of each figure).

some of the poorest fits to the observed OTL displacements in the north and up components of the $\mathrm{O}_{1}$ tide, but among the best fits in the east component. For the $\mathrm{M}_{\mathrm{f}}$ tide, all tide models perform similarly in the east component, with larger differences of up to about $0.1-0.2 \mathrm{~mm}$ in the north and up components. Although the regional ENPAC15 model outperforms other models in the north component of the $\mathrm{M}_{\mathrm{f}}$ harmonic, ENPAC15 yields relatively large residuals in the up component. The GOT4.10c suite of ocean-tide models does not include the $\mathrm{M}_{\mathrm{f}}$ harmonic, and is therefore not included in the bottom row of panels in Fig. 6. Residuals normalized by the amplitudes of the observed OTL response at each station are shown in the Supporting Information.

On the whole, no single ocean-tide model emerges as a preferred model in all three spatial components and for all three tidal harmonics. The TPXO9-Atlas model, which has local tide models integrated into a global solution, and the regional ENPAC15 model provide the best fits to the observed OTL displacements for the $\mathrm{M}_{2}$ harmonic. The FES2014b model yields relatively small residuals overall for the $\mathrm{O}_{1}$ and $\mathrm{M}_{\mathrm{f}}$ harmonics.

Direct comparisons between pairs of predicted OTL displacements that assume different ocean-tide models (Fig. S7) confirm that the largest $\mathrm{M}_{2}$ residuals consistently appear in the vicinity of Glacier Bay, where the ocean tides are known to be complicated due to narrow inlets and high energy dissipation through bottom friction (Inazu et al. 2009), as well as near the shoreline of the Gulf of Alaska and along the Aleutian Island chain (see Fig. 1a). The $\mathrm{O}_{1}$ and $\mathrm{M}_{\mathrm{f}}$ residuals are also relatively large near the Gulf of Alaska, Aleutian Islands and Bering Sea (Figs S8 and S9). Although the resolutions of ocean-tide models have improved markedly in recent years (Stammer et al. 2014), the models, to varying degrees, still cannot account for highly intricate coastal geometries with shoreline shapes varying on the order of a few kilometers or less.

\subsection{Network-coherent residual displacements}

We find that vector differences between predicted and observed OTL displacements are sometimes well correlated in amplitude and phase across the network (e.g. Fig. 2d), which suggests that an OTL displacement common to all stations could be isolated and removed from the residuals. We are primarily interested in identifying spatial variations in OTL residuals across Alaska because they are most relevant to revealing deficiencies in key forward-modelling assumptions, such as mismodelled ocean tides and solid-Earth structure, at the local to regional scale. Of lesser interest would be networkuniform residual displacements that may relate to long-wavelength errors in GPS processing and in the creation of ocean-tide models. 

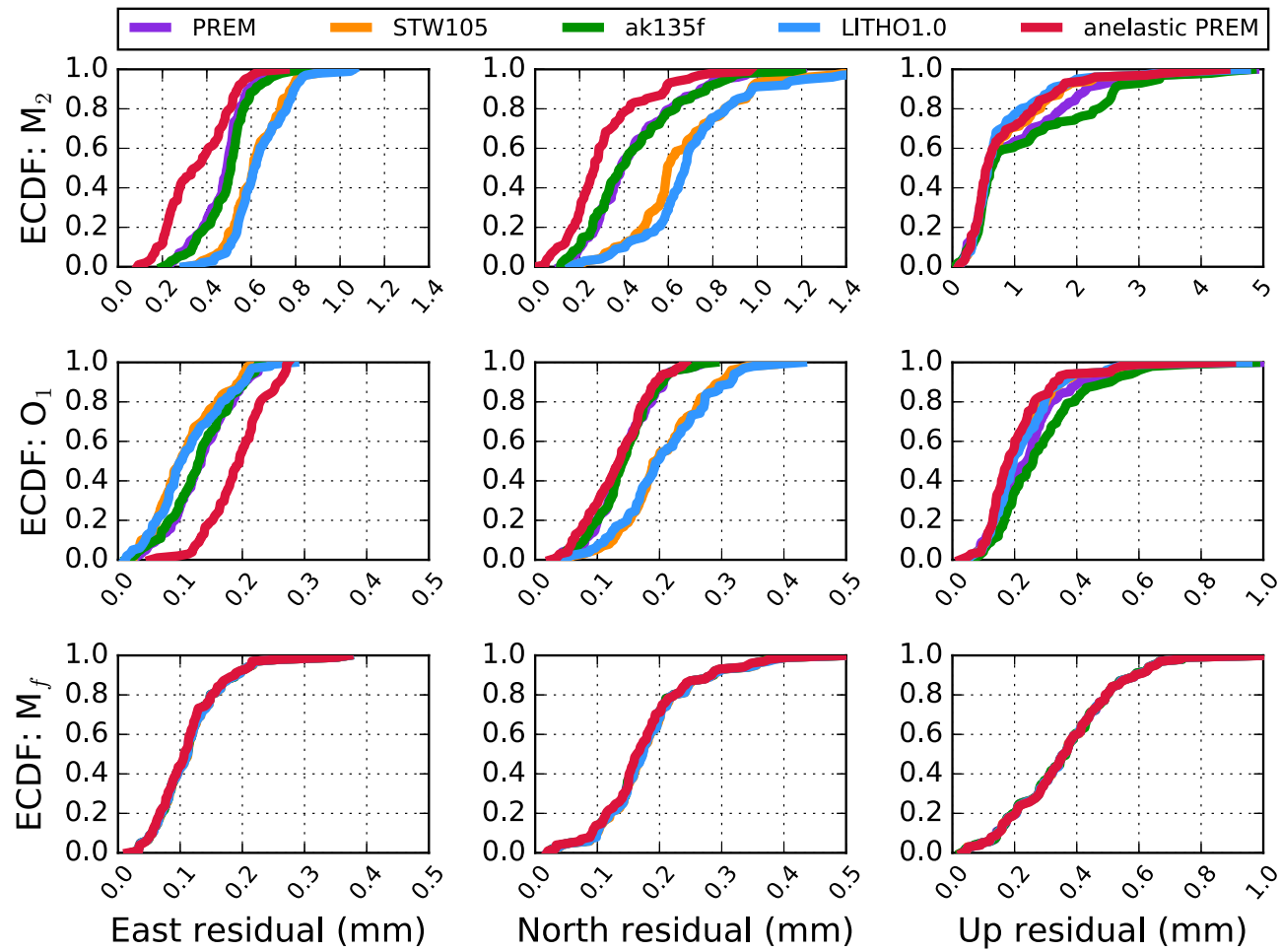

Figure 5. Empirical cumulative distribution functions (ECDFs) of residuals between predicted and observed OTL displacements for the $M_{2}, O_{1}$ and $M_{f}$ tidal harmonics. For each prediction, the ocean-tide model is held fixed (FES2014b) and the Earth model is varied (see legend at top). Each row depicts residuals for a different tidal harmonic (top: $\mathrm{M}_{2}$; middle: $\mathrm{O}_{1}$; bottom: $\mathrm{M}_{\mathrm{f}}$ ) and each column depicts residuals for a different spatial component (left: east; centre: north; right: up). Note that the scales of the $x$-axes vary by harmonic and spatial component.
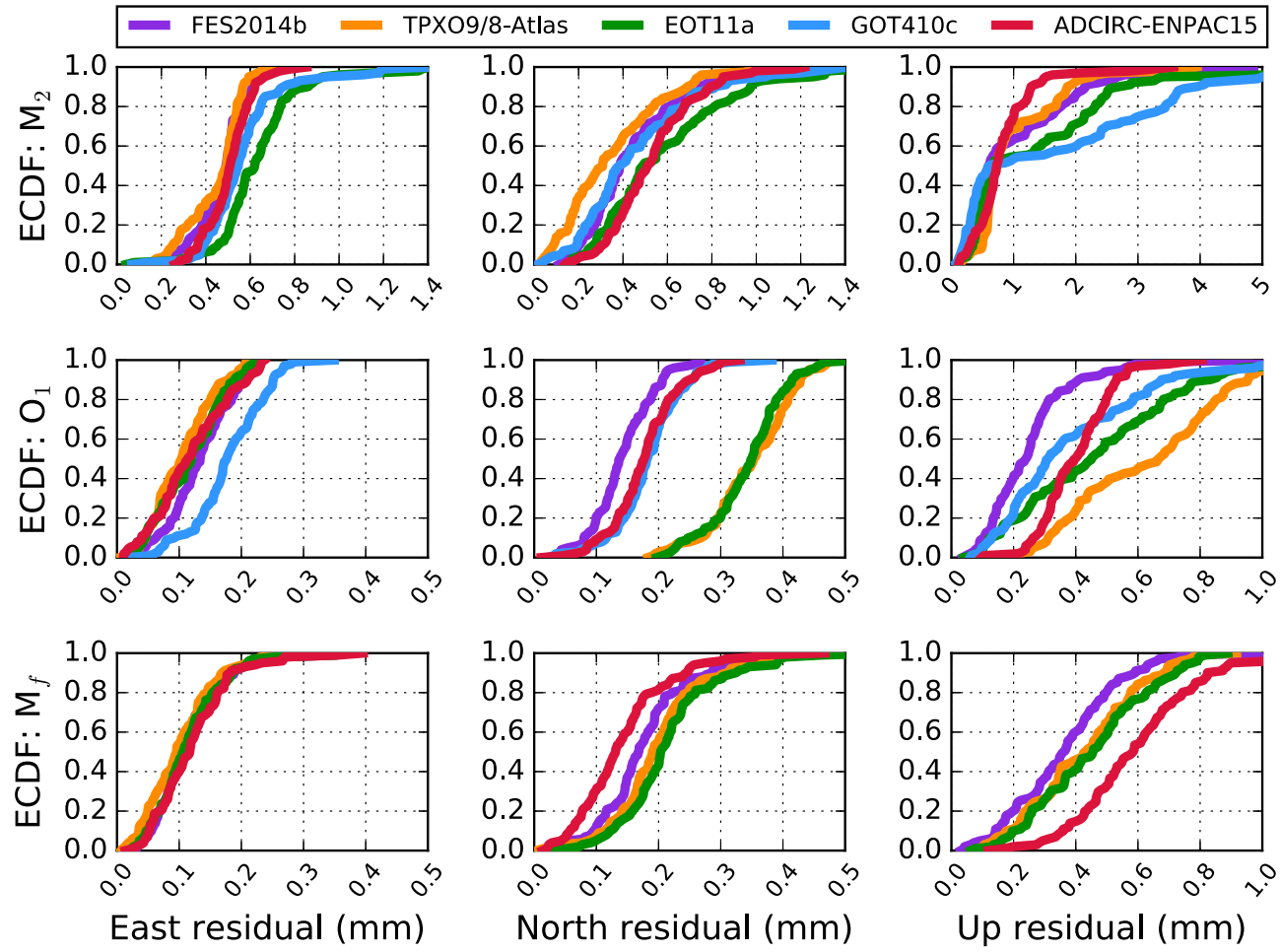

Figure 6. Empirical cumulative distribution functions (ECDFs) of residuals between predicted and observed OTL displacements for the $\mathrm{M}_{2}, \mathrm{O}_{1}$ and $\mathrm{M}_{\mathrm{f}}$ tidal harmonics. For each prediction, the Earth model is held fixed (PREM) and the ocean model is varied (see legend at top). Each row depicts residuals for a different tidal harmonic (top: $\mathrm{M}_{2}$; middle: $\mathrm{O}_{1}$; bottom: $\mathrm{M}_{\mathrm{f}}$ ) and each column depicts residuals for a different spatial component (left: east; centre: north; right: up). Note that the scales of the $x$-axes vary by harmonic and spatial component. For comparison, the scales of the axes are identical to those in Fig. 5 . 
For example, Martens et al. (2016b) showed that tide-model deficiencies in high-latitude regions due to sparse altimetry constraints, inaccuracies in the assumed uniform value for sea water density, reference-frame inconsistencies between predicted and observed OTL displacements, and reference-frame inconsistencies at various stages of the GPS data processing and tide-model development can contribute to a network-uniform harmonic displacement.

We refer to a network-uniform tidal-harmonic displacement as a 'harmonic common-mode' component (cf. Martens et al. 2016b), but caution that the harmonic common mode differs from a traditional common mode in GPS data analysis (e.g. some form of a network-averaged displacement time-series). We compute the harmonic common mode, separately for each spatial component and for each set of vector differences between predicted and observed displacements, by averaging independently the in-phase and quadrature components of the OTL residuals across the network.

Fig. 7 depicts residual displacements for several ocean-tide and Earth model combinations $\left(\mathrm{M}_{2}\right.$ harmonic). The left-hand column shows the full vector differences between predicted and observed OTL displacement at each station. The right-hand column reproduces the residuals, but with the harmonic common mode removed. In Fig. 7, we consider the FES2014b and ENPAC15 ocean-tide models and the LITHO1.0 and PREM Earth models; both Earth models are adjusted to account for anelastic dispersion in the upper mantle at the period of the $\mathrm{M}_{2}$ tide using estimates of $Q$ from ak135f and PREM, respectively. Removing the harmonic common mode can reduce the median residuals by several tenths of a millimeter. Even after removing the harmonic common mode, $\mathrm{M}_{2}$ residuals in Alaska exceed the observational uncertainties (Fig. 2c) and exhibit patterns of regional spatial coherency. We conclude that GPS measurement errors do not limit the ability to probe $\mathrm{M}_{2}$ OTL residuals for information about tide- and Earth-model deficiencies.

The PMEs in the northern part of Alaska (prior to removal of the harmonic common mode; Fig. 7, left-hand column) are consistently aligned toward the Bering Sea and Bering Strait, suggesting that the ocean-tide models in the Bering Sea and Bering Strait may contribute a dominant source of error. However, the semimajor axis orientations of the PMEs remain mostly consistent when adopting different ocean-tide models. After the harmonic common mode is removed, the choice of Earth model (i.e. anelastic LITHO1.0 versus anelastic PREM) is found to exert greater control on the orientations of the PMEs than the choice of ocean-tide model (i.e. ENPAC15 versus FES2014b). For other model combinations, however, the relative influence of the ocean model can exceed that of the Earth model (e.g. GOT4.10c and TPXO9-Atlas with ak135f and PREM; see also Figs 5 and 6).

Fig. 7(c) can be compared with Fig. 2(d) to see the effects of reducing the shear modulus in the upper mantle due to anelastic dispersion on the residual displacements for the $\mathrm{M}_{2}$ harmonic. We find that residuals are reduced in all three spatial components, suggesting that accounting for anelastic dispersion plays an important role in minimizing the misfit between predicted and observed OTL for the $\mathrm{M}_{2}$ harmonic in Alaska. Bos et al. (2015) also found an improved correlation between predicted and observed $\mathrm{M}_{2}$ OTL in western Europe after accounting for anelastic dispersion in the upper mantle, as did Wang et al. (2020) for GPS stations in eastern Asia.

Figs 8 and 9 show selected residual displacements for the $\mathrm{O}_{1}$ and $\mathrm{M}_{\mathrm{f}}$ harmonics, respectively. We again compare combinations of the FES2014b and ENPAC15 ocean-tide models with anelastic PREM and LITHO1.0 Earth models. Removing a harmonic common mode reduces the magnitude and improves the spatiotemporal consistency of residuals derived from different forward models, particularly for the $\mathrm{O}_{1}$ harmonic.

None of the forward-model combinations could reduce the $\mathrm{M}_{2}$ and $\mathrm{O}_{1}$ residuals below the estimated GPS observational uncertainties; most of the $\mathrm{M}_{\mathrm{f}}$ residuals also exceed estimated observational uncertainties. Furthermore, residuals for the $\mathrm{M}_{2}, \mathrm{O}_{1}$ and $\mathrm{M}_{\mathrm{f}}$ harmonics exhibit non-random spatial patterns, suggesting that the residuals might be used in future studies to further refine regional ocean-tide models and/or Earth-structure models. In generating predicted displacements, for example, we did not consider deviations from spherically symmetric structure. To explore 3-D variations in structure, which could better capture the complex tectonic and volcanic environments of Alaska ( $c f$. Khan \& Scherneck 2003), it is necessary to move beyond the load Green's function approach and to adopt fully numerical methods. We hypothesize that the influence of 3-D structure on OTL displacements may be significant at the current levels of modelling and observational precision, and evaluating sensitivities to $3-\mathrm{D}$ structure should be a priority in future studies.

\section{CONCLUSIONS}

We demonstrate that GPS can detect OTL deformation across Alaska in three frequency bands: $\mathrm{M}_{2}, \mathrm{O}_{1}$ and $\mathrm{M}_{\mathrm{f}}$. Even though the amplitude of the $\mathrm{M}_{\mathrm{f}}$ ocean tide reaches only a few centimeters in the Alaska region, residuals between predicted and observed $\mathrm{M}_{\mathrm{f}}$ displacements slightly exceed the estimated two-sigma observational uncertainties at most stations (e.g. Fig. 4). Estimated RMS observational uncertainties for the $\mathrm{M}_{\mathrm{f}}$ tide are $\pm 0.21 \mathrm{~mm}$ at the two-sigma level in the vertical component and $\pm 0.07 \mathrm{~mm}$ in the horizontal components (see Table S1 in the Supporting Information). Residual displacements vary depending on the forward model, but median vector differences between predicted and observed $\mathrm{M}_{\mathrm{f}}$ displacements generally range from 0.3 to $0.6 \mathrm{~mm}$ in the vertical component and $0.1-0.2 \mathrm{~mm}$ in the horizontal components (e.g. Figs 5 and 6).

Particularly for the larger $\mathrm{M}_{2}$ and $\mathrm{O}_{1}$ tides, the residuals mostly exceed the observational uncertainties by at least several-fold (e.g. Figs 2 and 3). Estimated RMS observational uncertainties for the $\mathrm{M}_{2}$ and $\mathrm{O}_{1}$ tides are remarkably small: $\pm 0.08 \mathrm{~mm}$ at the two-sigma level in the vertical component and $\pm 0.03 \mathrm{~mm}$ in the horizontal components. Median vector differences between predicted and observed $\mathrm{M}_{2}$ displacements generally range from $0.5-0.8 \mathrm{~mm}$ in the vertical component and $0.2-0.7 \mathrm{~mm}$ in the horizontal components. For the $\mathrm{O}_{1}$ tide, median vector differences range from $0.2-0.6 \mathrm{~mm}$ in the vertical component and $0.1-0.4 \mathrm{~mm}$ in the horizontal components. The ranges of residual amplitudes are consistent with recent OTL studies in western Europe (Bos et al. 2015) and South America (Martens et al. 2016b). Additional improvements in measurement precision would help to further reduce the observational uncertainties and residuals to better reveal deficiencies in the assumed Earth structure, particularly for small-amplitude, long-period tides.

We find that no ocean-tide and Earth model combination considered here emerges as the single preferred OTL model across all spatial components and tidal harmonics. Some forward models, however, perform better than others at reducing the misfit between predicted and observed OTL displacements on the whole. Accounting for the effects of anelastic dispersion in the upper mantle, for example, is effective at improving fits to the observed $\mathrm{M}_{2}$ displacements, as well as to the north and up components of the $\mathrm{O}_{1}$ tide. $\mathrm{Re}-$ cent studies of anelastic dispersion at tidal periods also found misfit reductions, but considered only the $\mathrm{M}_{2}$ harmonic (Bos et al. 2015; 


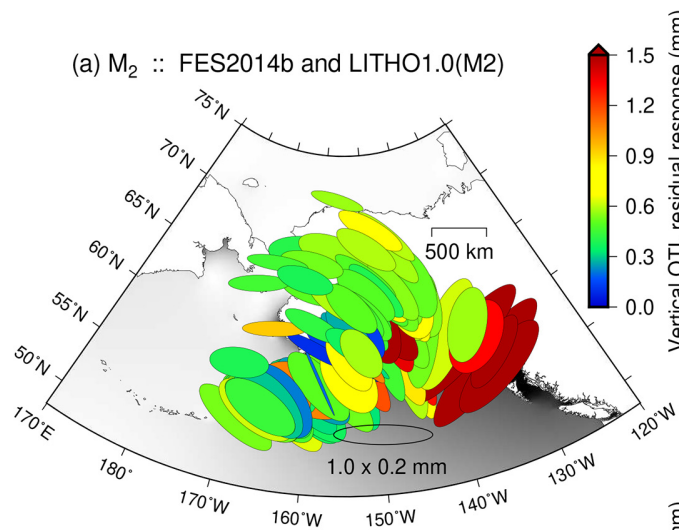

(c) $M_{2}::$ FES2014b and PREM(M2)

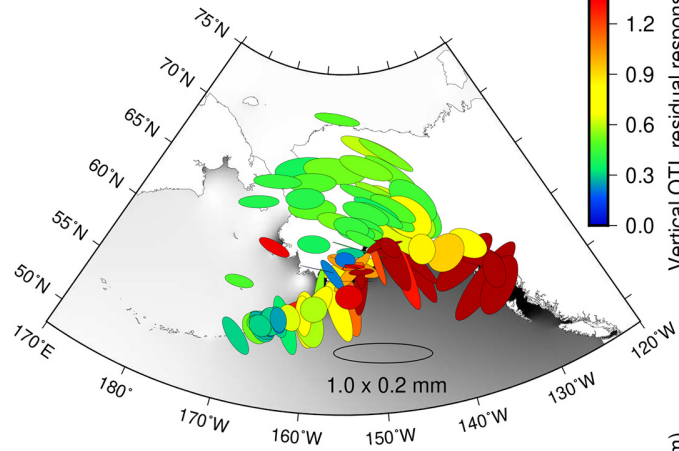

है

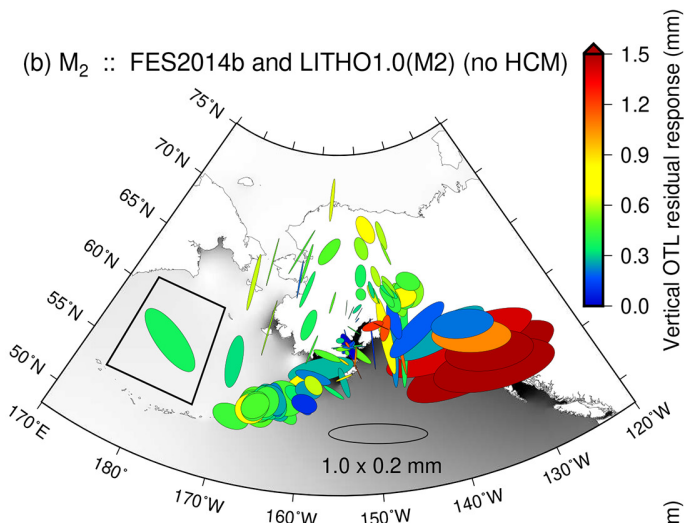

(d) $M_{2}::$ FES2014b and PREM(M2) (no HCM)

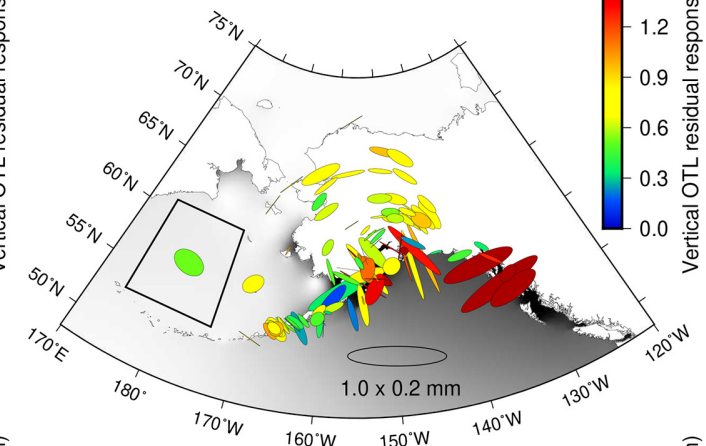

(e) $M_{2}$ :: ENPAC15 and LITHO1.0(M2)

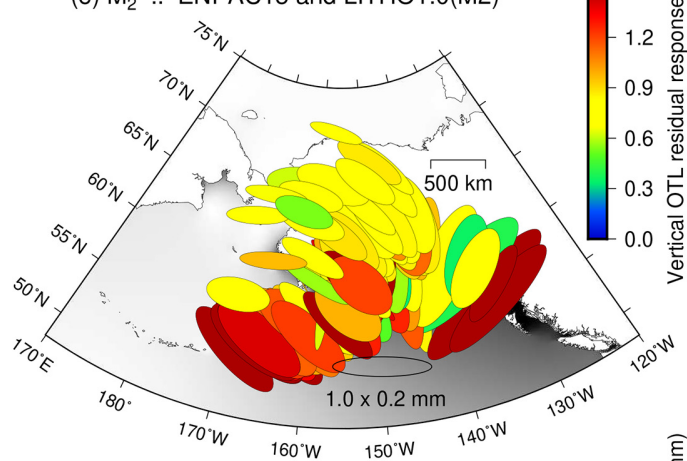

(g) $M_{2}$ :: ENPAC15 and PREM(M2)
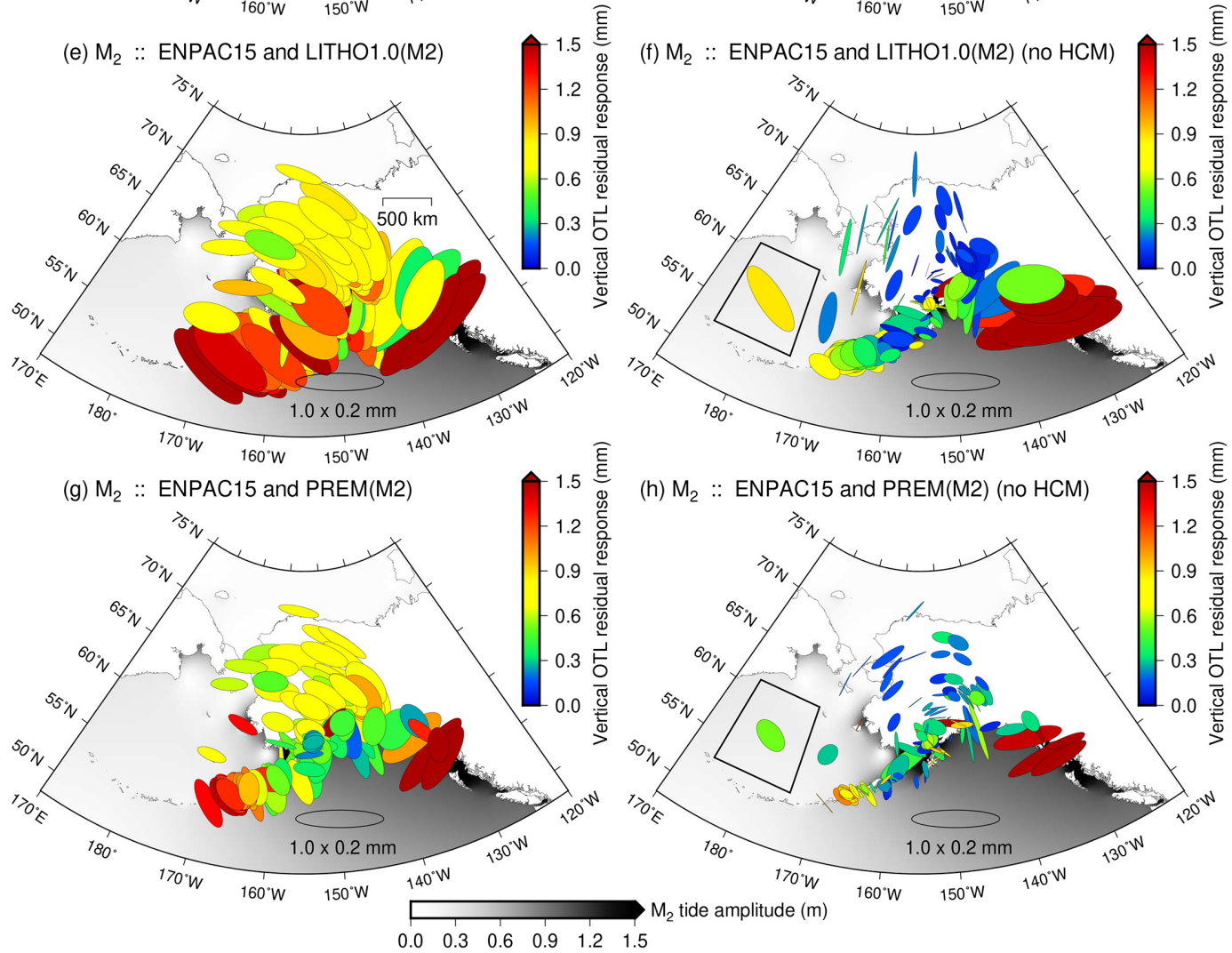

(h) $M_{2}$ :: ENPAC15 and PREM(M2) (no HCM)

Figure 7. Comparisons of $\mathrm{M}_{2}$ OTL residuals for four Earth- and tide-model combinations. The vector differences are shown by PMEs (see description in Fig. 2 caption). Residuals are shown both before (left-hand column) and after (right-hand column) a harmonic common mode (HCM) has been estimated and removed from the residuals. When a HCM has been removed, it is shown as a boxed PME in the lower left-hand corner of the figure. We consider the following model combinations: FES2014b and anelastic LITHO1.0 (a and b); FES2014b and anelastic PREM (c and d); ENPAC15 and anelastic LITHO1.0 (e and f); and ENPAC15 and anelastic PREM ( $\mathrm{g}$ and $\mathrm{h}$ ). The first panel in each pair (i.e. panels a, c, e and g) represents the residuals before the HCM is removed. The second panel in each pair (i.e. panels b, $d, f$ and $h$ ) represents the residuals after the HCM is removed. 


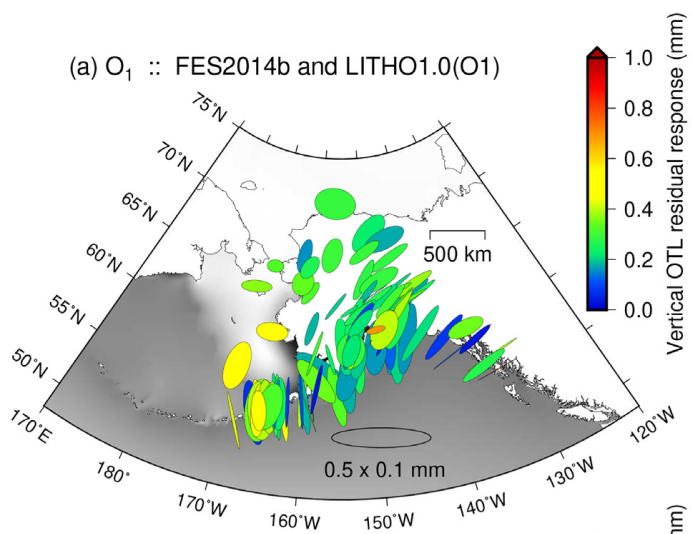

(c) $O_{1}::$ FES2014b and PREM(O1)

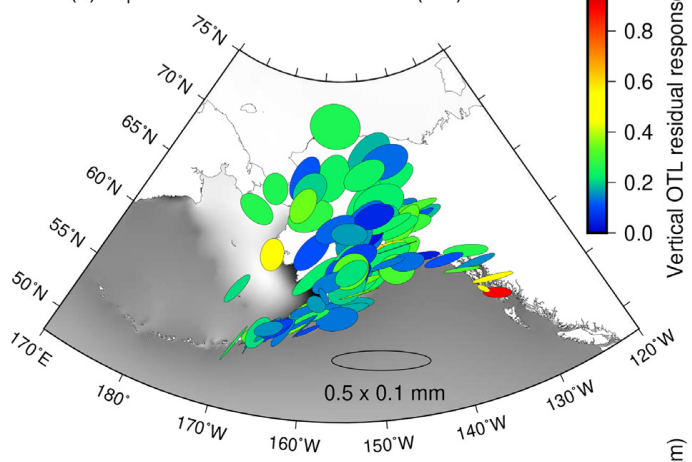

(e) $\mathrm{O}_{1}:$ : ENPAC15 and LITHO1.0(O1)



(g) $\mathrm{O}_{1}::$ ENPAC15 and PREM(O1)
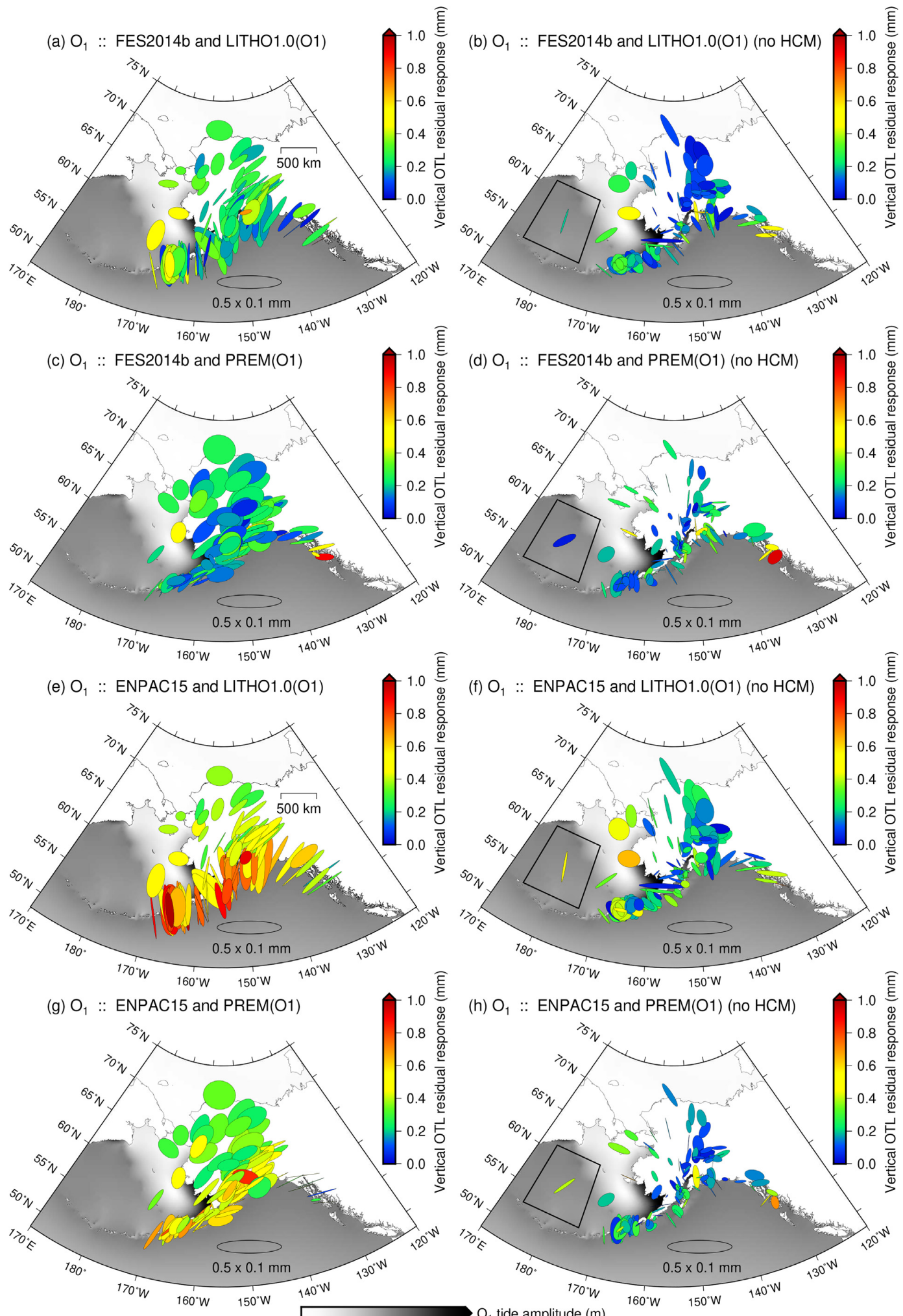

(d) $\mathrm{O}_{1}::$ FES2014b and PREM(O1) (no HCM)
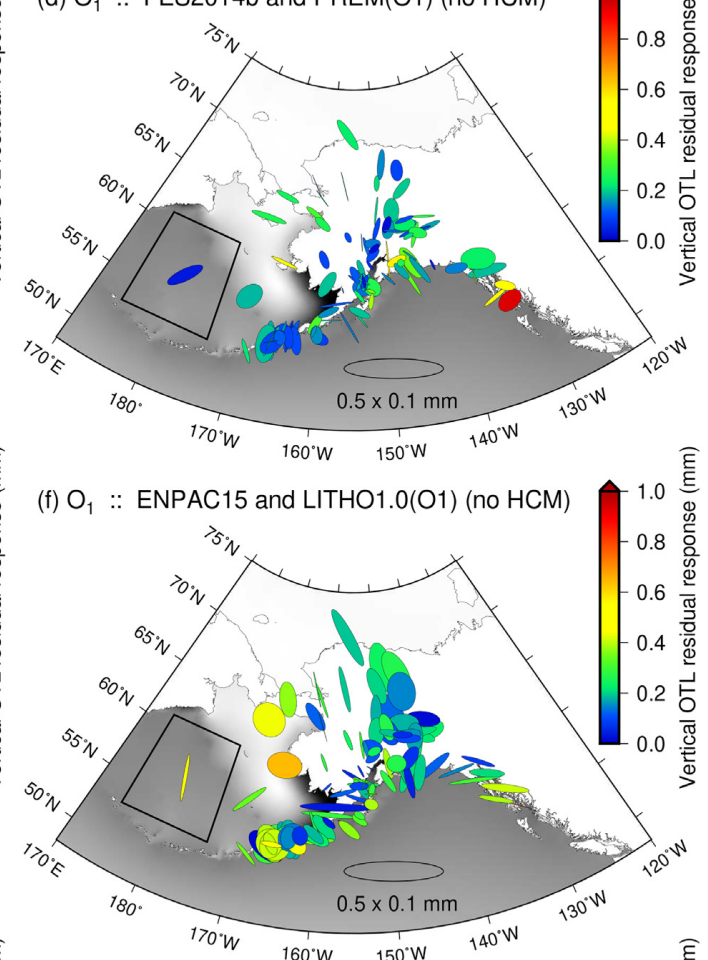

(h) $\mathrm{O}_{1}::$ ENPAC15 and PREM(O1) (no HCM)

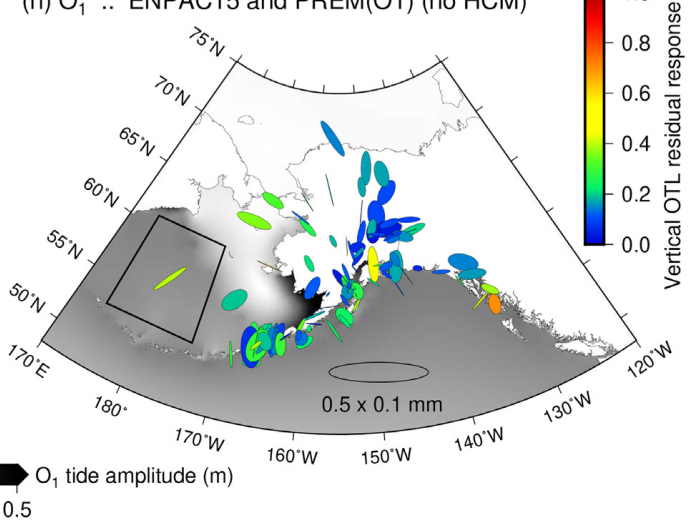

Figure 8. Same as Fig. 7, but for the $\mathrm{O}_{1}$ tidal harmonic. The Earth models have been adjusted to account for anelastic dispersion at the $\mathrm{O}_{1}$ tidal period.

Wang et al. 2020). Predictions of $\mathrm{M}_{\mathrm{f}}$ OTL displacements are mostly insensitive to the choice of Earth model as well as to adjustments for anelastic dispersion (at the level of microns). The east component of the $\mathrm{O}_{1}$ tide exhibits larger residuals after correcting for anelastic dispersion (Fig. 6). We find that the regionally customized LITHO1.0 model yields relatively good fits to the observed verticalcomponent data, albeit poorer fits to the horizontal components of the $\mathrm{M}_{2}$ and $\mathrm{O}_{1}$ tides. 

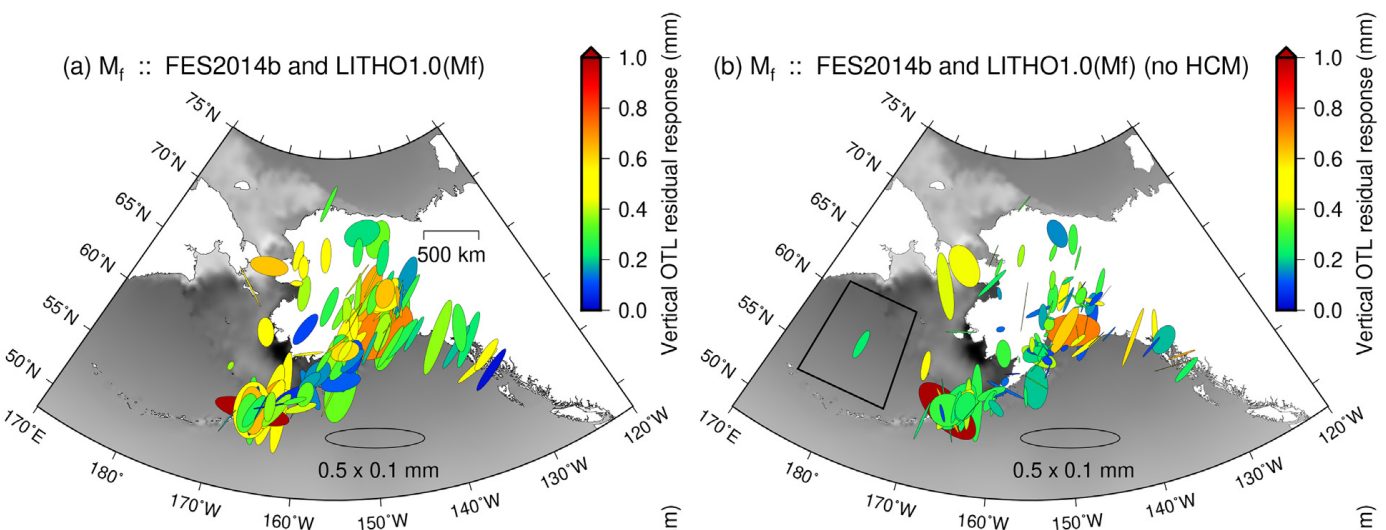

(c) $M_{f}::$ FES2014b and PREM(Mf)

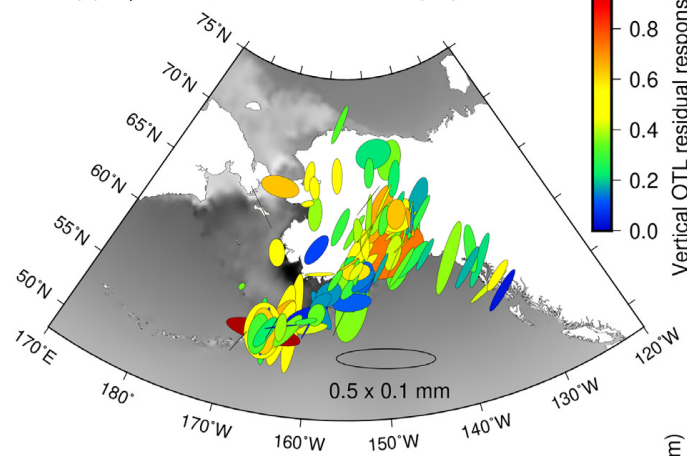

(d) $M_{f}::$ FES2014b and PREM(Mf) (no HCM)

(e) $M_{f}::$ ENPAC15 and LITHO1.0(Mf)

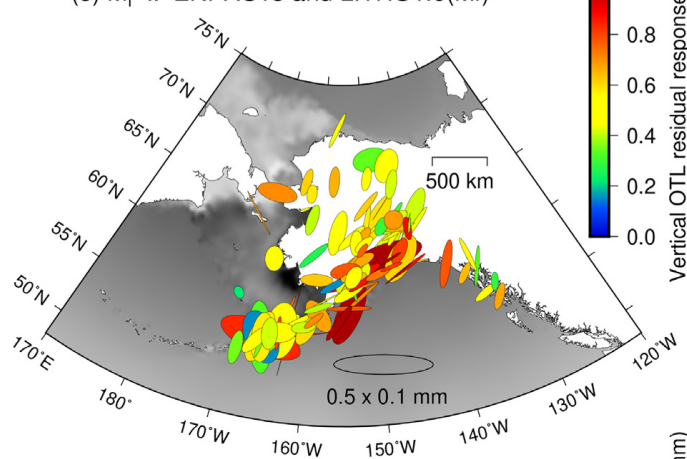

(g) $M_{f}::$ ENPAC15 and PREM(Mf)

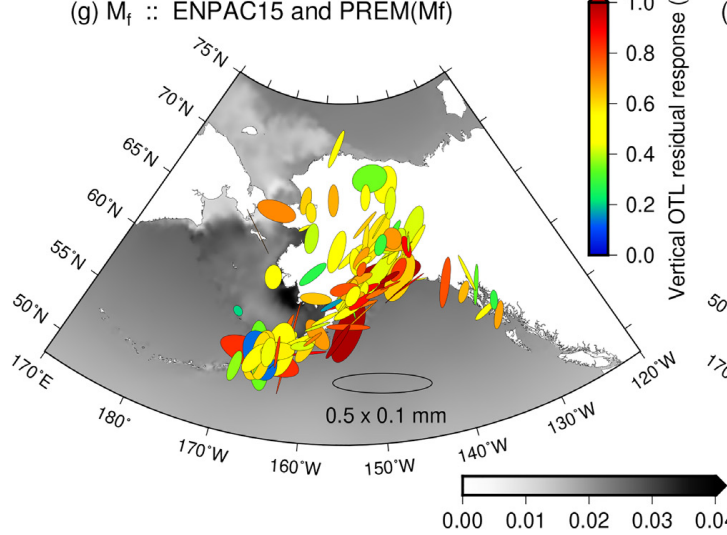

(h) $M_{f}::$ ENPAC15 and PREM(Mf) (no HCM)

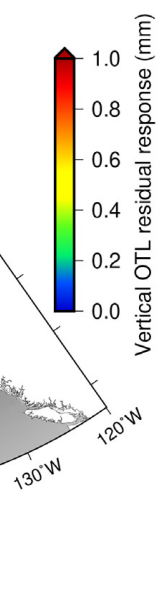

Figure 9. Same as Fig. 7, but for the $\mathrm{M}_{\mathrm{f}}$ tidal harmonic. The Earth models have been adjusted to account for anelastic dispersion at the $\mathrm{M}_{\mathrm{f}}$ tidal period.

Although predicted and GPS-observed OTL displacements for the $\mathrm{M}_{2}, \mathrm{O}_{1}$ and $\mathrm{M}_{\mathrm{f}}$ tides generally match at the level of $1 \mathrm{~mm}$ or better across Alaska regardless of the choice of ocean-tide or Earth model (Figs 5 and 6), larger residuals are found at coastal stations adjacent to large-amplitude tides (e.g. Fig. 7). Coastal stations are highly sensitive to the details of the ocean-tide model due to their proximity to the load (e.g. Martens et al. 2016a). Adopting a highresolution regional model for the ocean tides, ENPAC15, helps to 
reduce the observational residuals for the up component of the $\mathrm{M}_{2}$ harmonic (up to several $\mathrm{mm}$ ) and the north component of the $\mathrm{M}_{\mathrm{f}}$ harmonic (up to $0.1-0.2 \mathrm{~mm}$ ), but not for all components and harmonics (Fig. 6). On the whole, the FES2014b model performs well across all spatial components and tidal harmonics. The TPXO9Atlas model also provides relatively good fits to the observed data for the $\mathrm{M}_{2}$ harmonic. For the relatively small $\mathrm{M}_{\mathrm{f}}$ tide, the residuals reveal subtle, yet potentially detectable, discrepancies based on choice of ocean-tide model of about $0.1-0.2 \mathrm{~mm}$ (same order of magnitude as observational uncertainties).

Vector differences between predicted and observed OTL displacements commonly exhibit some uniformity in amplitude and phase across the network. To better characterize regional spatial variations in OTL response, we experiment with isolating and removing a harmonic common mode from the residual OTL displacements, which likely arises in part from long-wavelength errors associated with GPS processing and ocean-tide modelling ( $c f$. Martens et al. 2016b). After removing a harmonic common mode, median discrepancies between predicted (ENPAC15 and anelastic PREM) and observed OTL displacements are only $0.3 \mathrm{~mm}$ or less across all three spatial components and tidal-frequency bands (Figs 7-9h), which is close to the current limit of observational precision. Furthermore, the residuals reveal consistent spatial patterns, particularly for the $\mathrm{M}_{2}$ and $\mathrm{O}_{1}$ tides, that likely contain important information about local and regional inconsistencies with the assumed Earth structure and ocean-tide distribution, including deviations from spherically symmetric Earth structure.

\section{ACKNOWLEDGEMENTS}

The LoadDef software used to model load-induced deformation is available from Martens et al. (2019). Observed and predicted loadtide displacements for the Alaska PBO network (computed here) are provided in the Supporting Information. FES2014 was produced by Noveltis, Legos, and CLS and distributed by Aviso+, with support from CNES (https://www.aviso.altimetry.fr/). Figures were created using the Generic Mapping Tools (Wessel et al. 2013) and Matplotlib (Hunter et al. 2007). GPS data used in our study are available in open archives from UNAVCO (ftp://dataout.unavco.org/pub/rinex/obs). HRM processed the GPS data, performed the tidal harmonic analysis, generated the forward models of predicted tidal displacements, assessed uncertainties and residuals, produced the figures, and wrote the manuscript. MS provided important feedback on the interpretation of results and the written manuscript. HRM wishes to thank colleagues and staff at ETHZürich for their kind hospitality and engaging discussions during the summer and fall of 2019. We are grateful to two anonymous reviewers for their constructive and thorough reviews of the manuscript. We also extend a special thanks to Editor Duncan Agnew for his thoughtful reviews and fruitful discussions on tidal harmonic analysis, as well as to Luis Rivera (Université de Strasbourg) for his generous contributions to the load-tide modelling and interpretation of results. This material is based on work supported by the National Science Foundation under Grant No. 1925267 and the National Aeronautics and Space Administration under Grant No. NNX15AK40A. This material is also based on services provided by the GAGE Facility, operated by UNAVCO, Inc., with support from the National Science Foundation and the National Aeronautics and Space Administration under NSF Cooperative Agreement EAR-11261833.

\section{R EFER E N CES}

Agnew, D.C., 2015. Earth tides, in Treatise on Geophysics, 2nd edn, Vol. 3, pp. 151-178, ed. Schubert, G., Elsevier B.V.

Alterman, Z., Jarosch, H. \& Pekeris, C., 1959. Oscillations of the Earth, Proc. R. Soc. Lond., A, 252(1268), 80-95.

Amante, C. \& Eakins, B.W., 2009. ETOPO1 1 arc-minute global relief model: procedures, data sources and analysis, NOAA Technical Memorandum NESDIS NGDC-24, National Geophysical Data Center, Marine Geology and Geophysics Division, Boulder, Colorado.

Aster, R.C., Borchers, B. \& Thurber, C.H., 2013. Parameter Estimation and Inverse Problems, Academic Press.

Baker, T., 1984. Tidal deformations of the Earth, Sci. Prog. Oxford, 69, 197-233.

Baker, T., Curtis, D. \& Dodson, A., 1996. A new test of Earth tide models in central Europe, Geophys. Res. Lett., 23(24), 3559-3562.

Bar-Sever, Y.E., Kroger, P.M. \& Borjesson, J.A., 1998. Estimating horizontal gradients of tropospheric path delay with a single GPS receiver, $J$. geophys. Res., 103(B3), 5019-5035.

Bertiger, W., Desai, S.D., Haines, B., Harvey, N., Moore, A.W., Owen, S. \& Weiss, J.P., 2010. Single receiver phase ambiguity resolution with GPS data, J. Geod., 84(5), 327-337.

Blewitt, G., 2003. Self-consistency in reference frames, geocenter definition, and surface loading of the solid Earth, J. geophys. Res., 108(B2), 2103.

Boehm, J., Werl, B. \& Schuh, H., 2006. Troposphere mapping functions for GPS and very long baseline interferometry from European Centre for Medium-Range Weather Forecasts operational analysis data, J. geophys. Res., 111(B2), doi:10.1029/2005JB003629.

Bos, M.S., Penna, N.T., Baker, T.F. \& Clarke, P.J., 2015. Ocean tide loading displacements in western Europe. Part 2: GPS-observed anelastic dispersion in the asthenosphere, J. geophys. Res., 120(9), 6540-6557.

Carrère, L., Lyard, F., Cancet, M., Guillot, A. \& Roblou, L., 2012. FES2012: a new global tidal model taking taking advantage of nearly 20 years of altimetry, in Proceedings of meeting "20 Years of Altimetry", Venice.

Cartwright, D. \& Edden, A., 1973. Corrected tables of tidal harmonics, Geophys. J. R. astr. Soc., 33, 253-264.

Cartwright, D. \& Taylor, R., 1971. New computations of the tide-generating potential, Geophys. J. R. astr. Soc., 23, 45-74.

Dahlen, F. \& Tromp, J., 1998. Theoretical Global Seismology, Princeton Univ. Press.

Darwin, G.H., 1898. The Tides and Kindred Phenomena in the Solar System: The Substance of Lectures Delivered in 1897 at the Lowell Institute, Boston, Massachusetts, Houghton, Mifflin, \& Company.

Dziewonski, A.M. \& Anderson, D.L., 1981. Preliminary reference Earth model, Phys. Earth Planet. Inter., 25(4), 297-356.

Egbert, G.D. \& Erofeeva, S.Y., 2002. Efficient inverse modeling of barotropic ocean tides, J. Atmos. Oceanic Technol., 19(2), 183-204.

Egbert, G.D., Erofeeva, S.Y. \& Ray, R.D., 2010. Assimilation of altimetry data for nonlinear shallow-water tides: quarter-diurnal tides of the Northwest European Shelf, Cont. Shelf Res., 30(6), 668-679.

Farrell, W., 1972a. Deformation of the Earth by surface loads, Rev. Geophys., 10(3), 761-797.

Farrell, W., 1972b. Global calculations of tidal loading, Nature, 238(81), 43-44.

Foreman, M., 1977. Manual for tidal heights analysis and prediction, Pacific Mar. Sci. Rep., 77-10, 1-58.

Foreman, M., Cherniawsky, J. \& Ballantyne, V., 2009. Versatile harmonic tidal analysis: improvements and applications, J. Atmos. Oceanic Technol., 26(4), 806-817.

Fu, Y. \& Freymueller, J.T., 2013. Repeated large slow slip events at the southcentral Alaska subduction zone, Earth planet. Sci. Lett., 375, 303311.

Fu, Y., Freymueller, J.T. \& van Dam, T., 2012. The effect of using inconsistent ocean tidal loading models on GPS coordinate solutions, J. Geod., 86(6), 409-421.

Godin, G., 1972. The Analysis of Tides, University of Toronto Press.

Guo, J., Li, Y., Huang, Y., Deng, H., Xu, S. \& Ning, J., 2004. Green's function of the deformation of the Earth as a result of atmospheric loading, Geophys. J. Int., 159(1), 53-68. 
Herring, T.A., et al., 2016. Plate Boundary Observatory and related networks: GPS data analysis methods and geodetic products, Rev. Geophys., 54(4), 759-808.

Hunter, J.D., 2007. Matplotlib: A 2D graphics environment, Computing in Science \& Engineering, 9(3):90-95.

Inazu, D., Sato, T., Miura, S., Ohta, Y., Nakamura, K., Fujimoto, H., Larsen, C.F. \& Higuchi, T., 2009. Accurate ocean tide modeling in southeast Alaska and large tidal dissipation around Glacier Bay, J. Oceanogr., 65(3), 335-347.

Ito, T. \& Simons, M., 2011. Probing asthenospheric density, temperature, and elastic moduli below the western United States, Science, 332(6032), 947-951.

Kennett, B., Engdahl, E. \& Buland, R., 1995. Constraints on seismic velocities in the Earth from traveltimes, Geophys. J. Int., 122(1), 108-124.

Khan, S.A. \& Scherneck, H.-G., 2003. The M2 ocean tide loading wave in Alaska: vertical and horizontal displacements, modelled and observed, $J$. Geod., 77(3-4), 117-127.

Khan, S.A. \& Tscherning, C.C., 2001. Determination of semi-diurnal ocean tide loading constituents using GPS in Alaska, Geophys. Res. Lett., 28(11), 2249-2252.

King, M.A., Penna, N.T., Clarke, P.J. \& King, E.C., 2005. Validation of ocean tide models around Antarctica using onshore GPS and gravity data, J. geophys. Res., 110(B8), doi:10.1029/2004JB003390.

Kustowski, B., Ekström, G. \& Dziewoński, A., 2008. Anisotropic shearwave velocity structure of the Earth's mantle: a global model, J. geophys. Res., 113(B06306), doi:10.1029/2007JB005169

Latychev, K., Mitrovica, J.X., Ishii, M., Chan, N.-H. \& Davis, J.L., 2009. Body tides on a 3-D elastic earth: toward a tidal tomography, Earth Planet. Sci. Lett., 277(1-2), 86-90.

Lau, H.C., Mitrovica, J.X., Davis, J.L., Tromp, J., Yang, H.-Y. \& Al-Attar, D., 2017. Tidal tomography constrains Earth's deep-mantle buoyancy, Nature, 551(7680), 321.

Longman, I., 1962. A Green's function for determining the deformation of the Earth under surface mass loads: 1. Theory, J. Geophys. Res., 67(2), $845-850$.

Longman, I., 1963. A Green's function for determining the deformation of the Earth under surface mass loads: 2. Computations and numerical results, J. geophys. Res., 68(2), 485-496.

Lyard, F., Lefevre, F., Letellier, T. \& Francis, O., 2006. Modelling the global ocean tides: modern insights from FES2004, Ocean Dyn., 56(5-6), 394415

Martens, H.R., Rivera, L. \& Simons, M., 2019. LoadDef: A Python-based toolkit to model elastic deformation caused by surface mass loading on spherically symmetric bodies, Earth Space Sci., 6(2), 311-323.

Martens, H.R., Rivera, L., Simons, M. \& Ito, T., 2016a. The sensitivity of surface mass loading displacement response to perturbations in the elastic structure of the crust and mantle, J. geophys. Res., 121(5), 3911-3938.

Martens, H.R., Simons, M., Owen, S. \& Rivera, L., 2016b. Observations of ocean tidal load response in South America from sub-daily GPS positions, Geophys. J. Int., 205(3), 1637-1664.

Montagner, J.-P. \& Kennett, B., 1996. How to reconcile body-wave and normal-mode reference Earth models, Geophys. J. Int., 125(1), 229-248.

NiTime Developers, 2020. Nitime: time series analysis for neuroscience data, NiPy Library, v. 0.8.1, https://nipy .org/nitime.

Okubo, S. \& Saito, M., 1983. Partial derivative of Love numbers, Bull. Géod., 57(1), 167-179.

Pasyanos, M.E., Masters, T.G., Laske, G. \& Ma, Z., 2014. LITHO1.0: an updated crust and lithospheric model of the Earth, J. geophys. Res., 119(3), 2153-2173.

Pawlowicz, R., Beardsley, B. \& Lentz, S., 2002. Classical tidal harmonic analysis including error estimates in MATLAB using T_TIDE, Comput. Geosci., 28(8), 929-937.

Penna, N.T., Clarke, P.J., Bos, M.S. \& Baker, T.F., 2015. Ocean tide loading displacements in western Europe. Part 1: validation of kinematic GPS estimates, J. geophys. Res., 120(9), 6523-6539.

Petit, G. \& Luzum, B., 2010. IERS Technical Note No. 36, IERS Conventions (2010), International Earth Rotation and Reference Systems Service, Frankfurt, Germany.
Pugh, D. \& Woodworth, P., 2014. Sea-level Science: Understanding Tides, Surges, Tsunamis and Mean Sea-level Changes, Cambridge Univ. Press.

Ray, R., 2013. Precise comparisons of bottom-pressure and altimetric ocean tides, J. geophys. Res., 118(9), 4570-4584.

Ray, R.D., 1999. A global ocean tide model from TOPEX/POSEIDON altimetry: GOT99. 2., NASA Technical Memorandum 209478, National Aeronautics and Space Administration, Goddard Space Flight Center, Greenbelt, MD.

Sato, T., 2010. Importance of the ocean tide modeling of regional scale in the Earth tide study, in Marees Terrestres Bulletin D'Informations, Vol. 146, pp. 11 751-11 765, ed. Barriot, J.-P., International Center for Earth Tides.

Savcenko, R. \& Bosch, W., 2012. EOT11a-empirical ocean tide model from multi-mission satellite altimetry, DGFI Report, 89.

Stammer, D., et al., 2014. Accuracy assessment of global barotropic ocean tide models, Rev. Geophys., 52(3), 243-282.

Szpilka, C., Dresback, K., Kolar, R. \& Massey, T.C., 2018. Improvements for the Eastern North Pacific ADCIRC Tidal Database (ENPAC15), $J$. Mar. Sci. Eng., 6(4), 131.

Takeuchi, H. \& Saito, M., 1972. Seismic surface waves, in Methods in Computational Physics,, Vol. 11/Seismology: Surface Waves and Earth Oscillations, pp. 217-295, Academic Press.

Thomson, D.J., 2007. Jackknifing multitaper spectrum estimates, IEEE Signal Proc. Mag., 24(4), 20-30.

Wang, J., Penna, N.T., Clarke, P.J. \& Bos, M.S., 2020. Asthenospheric anelasticity effects on ocean tide loading around the East China Sea observed with GPS, Solid Earth, 11(1), 185-197.

Wessel, P., Smith, W.H.F., Scharroo, R., Luis, J. \& Wobbe, F. 2013. Generic Mapping Tools: Improved Version Released, EOS Trans. AGU, 94 (45), 409-410.

Yuan, L., Chao, B.F., Ding, X. \& Zhong, P., 2013. The tidal displacement field at Earth's surface determined using global GPS observations, $J$. geophys. Res., 118, 2618-2632.

Zumberge, J., Heflin, M., Jefferson, D., Watkins, M. \& Webb, F., 1997. Precise point positioning for the efficient and robust analysis of GPS data from large networks, J. geophys. Res., 102(B3), 5005-5017.

Zürn, W., Beaumont, C. \& Slichter, L.B., 1976. Gravity tides and ocean loading in southern Alaska, J. geophys. Res., 81(26), 4923-4932.

\section{SUPPORTING INFORMATION}

Supplementary data are available at $G J I$ online.

\section{Martens_Simons_2020_Alaska_OTL_SupportingInformation.p} df

Table S1. The quadratic means and medians of estimated two-sigma uncertainties associated with the observed load-tide displacements across the Alaska PBO network.

Figure S1. A comparison between Earth models used in our study. Figure S2. Residuals between predicted $\mathrm{M}_{2}$ OTL displacements derived from different Earth models.

Figure S3. Same as Fig. S2, but for the $\mathrm{O}_{1}$ harmonic.

Figure S4. Same as Fig. S2, but for the $\mathrm{M}_{\mathrm{f}}$ harmonic.

Figure S5. Global ocean-tide amplitudes and phases from the FES2014b suite of ocean-tide models.

Figure S6. The amplitude and phase of the regional ADCIRC/ENPAC15 ocean-tide model for the $\mathrm{M}_{2}$ harmonic, supplemented by the global FES2014b model outside the bounds of ENPAC15, in the eastern north Pacific Ocean.

Figure S7. Residuals between predicted $\mathrm{M}_{2}$ OTL displacements derived from different ocean-tide models.

Figure S8. Same as Fig. S7, but for the $\mathrm{O}_{1}$ harmonic.

Figure S9. Same as Fig. S7, but for the $\mathrm{M}_{\mathrm{f}}$ harmonic.

Figure S10. A timeline showing when stations in the Alaska PBO GPS network were active (first 33 stations alphabetically). 
Figure S11. Same as Fig. S10, but for the second set of 33 stations alphabetically.

Figure S12. Same as Fig. S10, but for the third set of 33 stations alphabetically.

Figure S13. Same as Fig. S10, but for the final set of stations alphabetically.

Figure S14. (a) Observed and (b) predicted OTL displacements at PBO GPS stations in Alaska for the $\mathrm{M}_{2}$ harmonic, zoomed in on the region surrounding Anchorage where there is a dense distribution of GPS stations. (c) Observational uncertainties and (d) residuals between predicted and observed OTL displacements.

Figure S15. Same as Fig. S14, but for the $\mathrm{O}_{1}$ tidal harmonic.

Figure S16. Same as Fig. S14, but for the $\mathrm{M}_{\mathrm{f}}$ tidal harmonic.

Figure S17. Vector differences between predicted OTL displacements computed using different forward-model assumptions (grid resolution and sea water density). In each case, we use PREM and FES2014b as Earth-structure and ocean-tide inputs, respectively.

Figure S18. Same as Fig. 5 in the main text, but normalized by the observed OTL amplitude at each station (separately for each harmonic and spatial component). The residuals are given as a percentage of the total observed OTL response.

Figure S19. Same as Fig. 6 in the main text, but normalized by the observed OTL amplitude at each station (separately for each harmonic and spatial component). The residuals are given as a percentage of the total observed OTL response.

Data set S1. GPS-observed surface displacements and estimated uncertainties for the following tidal harmonics: $\mathrm{M}_{2}, \mathrm{O}_{1}, \mathrm{M}_{\mathrm{f}}, \mathrm{M}_{4}$, $\mathrm{N}_{2}, \mathrm{~S}_{2}, \mathrm{~L}_{2}, \mathrm{P}_{1}, \mathrm{Q}_{1}, \mathrm{~S}_{1}, \mathrm{M}_{\mathrm{m}}, \mathrm{S}_{\mathrm{a}}, \mathrm{S}_{\mathrm{sa}}$.

Data set S2. Predicted displacements for the $\mathrm{M}_{2}, \mathrm{O}_{1}$, and $\mathrm{M}_{\mathrm{f}}$ tidal harmonics derived from various Earth- and ocean-model combinations.

Data set S3. Earth models used to compute predicted OTL displacements.

Data set S4. Vector differences between predicted and observed OTL displacements for multiple Earth- and ocean-model combinations.

Please note: Oxford University Press is not responsible for the content or functionality of any supporting materials supplied by the authors. Any queries (other than missing material) should be directed to the corresponding author for the paper. 